\title{
ASYMPTOTIC LAWS FOR GEODESIC HOMOLOGY ON HYPERBOLIC MANIFOLDS WITH CUSPS
}

\author{
By Martine Babillot \& Marc Peigné
}

To Martine's memory. The redaction of this paper has been overshadowed by Martine's death in July 2003. All the main ideas were worked out together, I have done my best to finish this paper.

M. Peigné

\begin{abstract}
We consider a large class of non compact hyperbolic manifolds $M=$ $\mathbb{H}^{n} / \Gamma$ with cusps and we prove that the winding process $\left(Y_{t}\right)$ generated by a closed 1 -form supported on a neighborhood of a cusp $\mathcal{C}$, satisfies a limit theorem, with an asymptotic stable law and a renormalising factor depending only on the rank of the cusp $\mathcal{C}$ and the Poincaré exponent $\delta$ of $\Gamma$. No assumption on the value of $\delta$ is required and this theorem generalises previous results due to Y. Guivarc'h, Y. Le Jan, J. Franchi and N. Enriquez.
\end{abstract}

Texte reçu le 20 septembre 2004, accepté le 1er février 2005.

Martine Babillot, MAPMO, Fédération Poisson, Université d'Orléans, BP 6759, 45067 OrlŐéans Cedex (France).

Marc Peigné, Laboratoire de Mathématiques et Physique Théorique, Fédération Poisson, Université François Rabelais de Tours, Parc de Grandmont, 37200 Tours (France).

E-mail : peigne@univ-tours.fr

2000 Mathematics Subject Classification. - 58F17, 58F20, 20H10.

Key words and phrases. - Geodesic flow, asymptotic winding, hyperbolic manifolds, central limit theorem, stable law, transfer operator. 
RÉSUMÉ (Lois stables et flot géodésique sur des variétés non compactes à courbure négative)

Nous considérons une large classe de variétés hyperboliques non-compactes $M=$ $\mathbb{H}^{n} / \Gamma$ possédant des cusps et nous démontrons que le processus $\left(Y_{t}\right)$ engendré par une forme fermée portée par un voisinage d'un cusp $\mathcal{C}$ converge en loi vers une loi stable; la loi limite et le facteur de renormalisation dépendent de la nature du cusp et de l'exposant de Poincaré $\delta$ du groupe $\Gamma$. Aucune restriction sur la valeur de $\delta$ n'est imposée et cet article généralise ainsi toute une série de résultats dus à Y. Guivarc'h, Y. Le Jan, J. Franchi et N. Enriquez.

\section{Introduction.}

Sinai's observation that "dynamical systems generated by geodesic flows of negatively curved manifolds have a structure analogous to that off dynamical systems generated by stochastic processes" lead him to the proof of a Central Limit Theorem for certain additive functionals of the geodesic flow on a compact hyperbolic manifold $M$. A typical example of such a functional, due to Gel'fand and Pyateckii-Shapiro [15], is the "winding process", that is the process generated by a closed 1 -form on $M$.

Consider now a (not necessarily compact) hyperbolic manifold $M=\mathbb{H} / \Gamma$ where $\Gamma$ is a torsion-free group acting isometrically and properly discontinuously on the $n$-dimensional hyperbolic space $\mathbb{H}$. When $\Gamma$ is geometrically finite, the manifold $M$ is the union of a compact core and finitely many ends, some of which are funnels, and the other ones are cusps. If a geodesic enters a funnel, it goes to infinity without ever returning to the compact core. On the contrary, a typical geodesic entering a cusp does come back in the compact core infinitely often and thus belongs to the non-wandering set of the geodesic flow. These excursions in the cusps are related to diophantine approximation in number theory when $\Gamma$ is arithmetic (see [29], [36]), and in general, they describe how well can be approximated boundary points by parabolic ones (see [35]).

From the dynamical point of view, these excursions are now responsible for the non-uniform hyperbolicity of the geodesic flow, and it is therefore unclear which stochastic properties still hold. In this paper, we shall study the winding process around a cusp $\mathcal{C}$ of the manifold. More precisely, we fix a 1-form $\omega$ supported and closed on a neighborhood of $\mathcal{C}$. For a vector $v$ in the unit tangent bundle $T^{1} M$ of $M$, denote by $\left[v_{0}, v_{t}\right]$ the geodesic path of length $t$ on the geodesic starting at $v$. We get an additive functional of the geodesic flow by considering the process

$$
Y_{t}(v)=\int_{\left[v_{0}, v_{t}\right]} \omega
$$

TOME $134-2006-\mathrm{N}^{\mathrm{O}} 1$ 
and we are interested in the stochastic behavior of $\left(Y_{t}\right)$ with respect to some invariant measure $m$ of the geodesic flow. In particular, we shall say that $\left(Y_{t}, m\right)$ satisfies a limit theorem with renormalising factor $d(t)$ if there exists a probability measure $\pi$ on $\mathbb{R}$ such that for every real number a

$$
m\left\{v \in T^{1} M ; \frac{1}{d(t)} Y_{t}(v) \geq a\right\} \text { converges to } \pi(a,+\infty) \text { as } t \rightarrow \infty \text {. }
$$

One gets the classical Central Limit Theorem when $d(t)=\sqrt{t}$ and $\pi$ is the Gauss distribution. Otherwise, one looks either for $d(t)=\sqrt{t \log t}$ and $\pi$ the Gauss distribution, or for $d(t)$ of the form $t^{1 / \alpha}$ for some $\left.\alpha \in\right] 0,2[$, and $\pi$ a stable law of index $\alpha$.

For finite volume hyperbolic manifolds, the process $\left(Y_{t}\right)$ is known to satisfy a limit theorem with respect to the Liouville measure $m$. The case of the modular surface $M=\mathbb{H}^{2} / \mathrm{SL}(2, \mathbb{Z})$ has been worked out by Y. Guivarc'h and Y. Le Jan [18]: the winding of a typical geodesic satisfies a limit theorem with renormalisation factor $t$ and a Cauchy limit law ( $\alpha=1$ in this case). Further works rely on the comparaison between Brownian paths and geodesics, a method introduced by Le Jan [25]. This was used by Enriquez \& Le Jan [13] to extend the previous result to any hyperbolic surface and by Franchi [14] for 3dimensional manifolds; in this last case, the normalising factor becomes $\sqrt{t \log t}$, with a normal limit law. In higher dimension, the Central Limit Theorem holds since in this case the form $\omega$ is square integrable with respect to the Liouville measure.

For hyperbolic manifolds with infinite volume, the asymptotic behavior of $\left(Y_{t}\right)$ is still quite open. In this context, the natural probability measure to look at is the Patterson-Sullivan measure $m$ on $T^{1} M$ when it is finite, since in this case it is the unique measure of maximal entropy; in particular, it gives 0 -measure to the wandering set of the geodesic flow, and coincides with the Liouville measure when $M$ has finite volume. Let us call a cusp neutral if $\left(Y_{t}, m\right)$ satisfies the Central Limit Theorem, and influential if $\left(Y_{t}, m\right)$ satisfies a limit theorem with renormalising factor $d(t) \gg \sqrt{t}$ as $t \rightarrow+\infty$. From the series of work mentioned above, we see that for finite volume manifolds, all cusps are influential in dimension 2 and 3, and become neutral in higher dimension.

Our main observation here will be that this dichotomy on the dimension does not hold anymore for general hyperbolic manifolds. For a specific class of manifolds, we discover that the main role is played by the rank of the cusp $\mathcal{C}$, that is the rank of a maximal free abelian subgroup contained in its fundamental group $P=\pi_{1}(\mathcal{C})$.

Our main result concerns a restrictive class of Kleinian groups. Let us first introduce a definition: we say that finitely many Kleinian groups $\Gamma_{1}, \ldots, \Gamma_{L}$ are in Schottky position if there exist non-empty disjoint closed sets $F_{1}, \ldots, F_{L}$ in the boundary $\mathcal{S}^{n-1}$ of $\mathbb{H}^{n}$ such that $\Gamma_{i}^{*}\left(\mathcal{S}^{n-1}-F_{i}\right) \subset F_{i}$ for any $i \in\{1, \ldots, L\}$ 
(where the notation $\Gamma_{i}^{*}$ stands for $\Gamma_{i}-\{\operatorname{Id}\}$ ). The group $\Gamma$ generated by $P$ and $G$ is called the Schottky product of $P$ and $G$, and $P$ is called a Schottky factor of $\Gamma$.

We will say that a Schottky product group $\Gamma$ satisfies the critical gap hypothesis if its Poincaré exponant $\delta$ is strictly greater than the one of each subgroup $\Gamma_{i}$. For such a group $\Gamma$, the Patterson Sullivan measure $m$ on $T^{1}\left(\mathbb{H}^{n} / \Gamma\right)$ is finite (see [30]), and it is the unique measure of maximal entropy for the geodesic flow restricted to its non wandering set (see [27]); it will thus be the initial distribution for the processes we will consider since, roughly speaking, it caries most of the information about the stochastic behavior of the geodesic flow.

Note that classical Schottky groups (see for instance [26] for a definition) are Schottky products, with each $\Gamma_{i} \simeq \mathbb{Z}$ and that the critical gap hypothesis is automatically satisfied in this case [3].

We can now state the

MAIN TheOREM. - Let $\Gamma$ be a Schottky product of subgroups $\Gamma_{1}, \ldots, \Gamma_{L}$ of Iso $\left(\mathbb{H}^{n}\right)$, satisfying the critical gap hypothesis. Assume that one of the Schottky factors of $\Gamma$ is a parabolic group $P$ of rank $k$ and denote by $\mathcal{C}$ the cusp of $\mathbb{H}^{n} / \Gamma$ associated with $P$. Let $m$ be the Patterson-Sullivan measure on $T^{1}\left(\mathbb{H}^{n} / \Gamma\right)$.

For a closed 1-form $\omega$ supported on a neighbourhood of $\mathcal{C}$, the corresponding process $\left(Y_{t}, m\right)$ satisfies a limit theorem. The renormalising factor and the limit law depend on the values of the parameter $\alpha:=2 \delta-k$ as follows:

- if $\alpha<2, \quad d(t)=t^{1 / \alpha}$ and the limit law is a stable law of index $\alpha$;

- if $\alpha=2, d(t)=\sqrt{t \log t}$ and the limit law is a normal law;

- if $\alpha>2, \quad d(t)=\sqrt{t}$ and the limit law is a normal law.

Thus, we see that a cusp becomes influential if its rank is sufficiently big with respect to the Hausdorff dimension of the limit set $(k \geq 2 \delta-2)$. It should be noted that this condition extends the previous dichotomy for finite volume manifolds. Indeed, in this case, all cusps have maximal rank $k=n-1$ and the limit set is the whole sphere $\mathcal{S}^{n-1}$; thus, writing $n-1 \geq 2(n-1)-2$, we recover the previous condition $n \leq 3$.

Observe that if $\bar{k}$ is the maximal rank of the cusps in $M$, only cusps of rank $\bar{k}$ and $\bar{k}-1$ may become influential since, by Beardon's result [3], one knows that $\delta$ always satisfies the inequality $2 \delta>\bar{k}$.

We believe that this result might be true in particular for all hyperbolic manifolds whose Patterson-Sullivan measure is finite and in particular for all geometrically finite manifolds; this is partly confirmed by a recent result of Enriquez, Franchi \& Le Jan [12] who study the case of a manifold with a cusp of rank $n-1$ under the additional assumption that $2 \delta-(n-1)>1$.

TOME $134-2006-\mathrm{N}^{\mathrm{O}} 1$ 
To prove the Main Theorem, we first have to establish a classical Central Limit Therorem for this class of manifolds. This result can be stated in fact in the case of variable pinched curvature, we have the:

THEOREM III.5. - Let $X$ be a Hadamard manifold of pinched strictly negative curvature and $\Gamma=\Gamma_{1} * \cdots * \Gamma_{L}$ be a Schottky product of Kleinian groups acting on $X$ and satisfying the critical gap hypothesis. Let $M=X / \Gamma$ be the quotient manifold and $m$ the Patterson-Sullivan probability measure on $T^{1} M$. For any bounded and Hölder function $\Phi: T^{1} M \rightarrow \mathbb{R}$, the quantity

$$
\int_{T^{1} M} \frac{1}{t}\left(\int_{0}^{t}\left(\Phi\left(g_{s} v\right)-m(\Phi)\right) \mathrm{d} s\right)^{2} m(\mathrm{~d} v)
$$

converges to a constant $\sigma_{\Phi}^{2}$. One has $\sigma_{\Phi}^{2}=0$ if and only if $\Phi$ is a coboundary, i.e. if $\Phi$ is the derivate in the direction of the flow of some Borel function defined on $T^{1} M$.

- When $\sigma_{\Phi}^{2} \neq 0$, the process $X_{t}(v)=\int_{0}^{t} \Phi\left(g_{s} v\right) \mathrm{d} s$ satisfies the Central Limit Theorem: for any $a \in \mathbb{R}$

$$
m\left\{v: \frac{X_{t}(v)-t m(\Phi)}{\sigma_{\Phi} \sqrt{t}}>a\right\} \longrightarrow \pi([a,+\infty[)
$$

when $t \rightarrow+\infty$, where $\pi$ is a standard Gaussian law $\mathcal{N}(0,1)$ on $\mathbb{R}$.

- When $\sigma_{\Phi}^{2}=0$, the process $\left(\left(X_{t}-t m(\Phi)\right) / \sqrt{t}\right)_{t}$ tends to 0 in probability.

This theorem extends many previous results; let us cite for instance [34] (without the precise normalisation in $\sqrt{t}$ ), [32] (for compact manifolds in the variable curvature case), [25] (for hyperbolic manifolds of finite volume) and more recently [7] (with a martingale argument which can be applied in weakly hyperbolic situations [24]). Let us emphasize that the above simple expression of the asymptotic variance $\sigma_{\Phi}^{2}$ is obtained in a very elegant way in [7]; nevertheless, the proof given here simplifies Ratner's argument and does not require any speed of mixing of the geodesic flow, as in [7].

We can extend the Main Theorem in some others directions. First, one can fix a family $\left\{\omega_{1}, \ldots, \omega_{\kappa}\right\}$ of 1 -forms with support on a neighborhood of $\mathcal{C}$ which represents a basis of $H^{1}(\mathcal{C} ; \mathbb{R})$. Note that the homological rank $\kappa$ of the parabolic group $P$ may be smaller than its rank $k$ if $P$ is not abelian. We get therefore a multi-dimensional process $Y_{t}=\left(Y_{t}^{1}, \ldots, Y_{t}^{\kappa}\right)$ as above. This process has a limiting behavior and that the limit law is $\kappa$-dimensional stable, or Gaussian according to $\alpha<2$ or $\alpha \geq 2$.

We shall also see that the processes $X_{t}$ and $Y_{t}$ becomes asymptotically independent when the cusp $\mathcal{C}$ is influential, i.e. $\alpha<2$. An extension of this property is the following: one might consider all the cusps $\mathcal{C}_{1}, \ldots \mathcal{C}_{p}$ of the manifold simultaneously, and prove that the different processes built over the different cusps become asymptotically independent under $m$. 
Our approach to this problem, like in the original papers by M. Ratner [32] and Y. Guivarc'h \& Y. Le Jan [18], goes through a representation of the geodesic flow as a special flow over a dynamical system $(\Lambda, T)$ with a ceiling function $\ell$ (see for instance [2] for a very close set-up). The map $T$, called sometime the Nielsen map, is expanding on the subset $\Lambda$ of the limit set of $\Gamma$ which allows us to use transfer operator methods. The first main difficulty here is that the alphabet we use is not finite, but countable. Thus, our transformation $T$ can be thought as a distant cousin of the Gauss map which appears in the coding of the geodesic flow on the modular surface via continued fraction expansions (see [1], [6], [33]). Another difficulty relies on the fact that the ceiling function is unbounded; the approximation of the processes $X_{t}$ and $Y_{t}$ and the expression of the variance in term of the geodesic flow is thus technically much more difficult than in M. Ratner's original work.

\section{Schottky products and their limit sets}

When $\Gamma$ is a classical Schottky group generated by hyperbolic isometries, Bowen's [5] important work leads to a symbolic model for the geodesic flow on the (infinite volume) hyperbolic manifold $M$ with fundamental group $\Gamma$. This flow is bi-Lipschitz equivalent to a special flow over a subshift of finite type, whose expanding factor is conjugated with a geometric map $T$ on the limit set of $\Gamma$ : the Nielsen map. Thus, it was possible to study the flow using symbolic dynamics and thermodynamical formalism (see [23], [28]). When the group $\Gamma$ is a Schottky group containing parabolic transformations, and therefore when the manifold $M$ has cusps, this construction can be extended almost verbatim, but presents two major problems: the equivalence between the Nielsen map $T$ and the symbolic shift is neither onto, nor Lipschitz, and the map $T$ is no longer strictly expanding. Hence, the methods of symbolic dynamics could not be applied directly. After Series' work [33], a construction was proposed in [9] which lead to a coding (up to a set of 0-measure for the relevant measure), of the geodesic flow as a suspension over a subshift with countable alphabet. This construction was applied to count closed geodesics (see [2], [9]).

This section is devoted to an extension of the previous construction in the variable curvature case. After fixing some notation, we consider what we call the "Schottky product" of simpler groups $\Gamma_{i}$. If one assumes that the critical gap hypothesis holds (which occurs in particular when the groups $\Gamma_{i}$ are divergent, see [30]), then the resulting group $\Gamma$ is also divergent, and "most" of the limit set of $\Gamma$ is bi-Lipschitz equivalent with a subshift of finite type in the symbolic space $\Sigma=\mathcal{A}^{\mathbb{Z}}$ with countable alphabet $\mathcal{A}=\bigcup \Gamma_{i}^{\star}$. This construction will be used in the next section to code the geodesic flow on $T^{1} M$.

II-a. Notations. - We refer to [4] and [22] for the differents tools which are explained here and for further references. Let $X$ be a Hadamard manifold of

TOME $134-2006-\mathrm{N}^{\mathrm{O}} 1$ 
pinched negative curvature $K \leq-1$ endowed with a distance $d$. Its boundary at infinity $\partial X$ is the set of equivalence classes of asymptotic geodesic rays.

For a boundary point $x$ and two points $\mathbf{x}, \mathbf{y}$ in $X$, the limit $B_{x}(\mathbf{x}, \mathbf{y})$ of

$$
d(\mathbf{x}, \mathbf{z})-d(\mathbf{y}, \mathbf{z})
$$

as $\mathbf{z}$ goes to $x$ is called the Busemann cocycle; it represents the algebraic distance between the two horospheres centered at $x$ and passing through $\mathbf{x}$ and $\mathbf{y}$ respectively. If $x, y \in \partial X$ and $\mathbf{o}$ is a fixed "origin" in $X$, the "Gromov product" of $x$ and $y$ seen from $\mathbf{o}$ is defined by

$$
(x \mid y)_{\mathbf{o}}=\frac{1}{2}\left(B_{x}(\mathbf{o}, \mathbf{z})+B_{y}(\mathbf{o}, \mathbf{z})\right)
$$

and does not depend on the point $\mathbf{z}$ on the geodesic $(x y)$; the curvature being bounded from above by -1 , the quantity $\exp \left(-(x \mid y)_{\mathbf{o}}\right)$ is a distance on $\partial X$, it coincides with the euclidean distance on $\mathcal{S}^{n-1}$ when $X$ is the $n$-dimensional hyperbolic space, and will thus be denoted by $|x-y|$.

The group Iso $(X)$ of orientation-preserving isometries of $X$ acts continuously on $\partial X$ by conformal transformations. The conformal factor of an isometry $\gamma$ at the point $x \in \partial X$ is given by the formula

$$
\left|\gamma^{\prime}(x)\right|=\mathrm{e}^{-B_{x}\left(\gamma^{-1} \cdot \mathbf{o}, \mathbf{o}\right)} .
$$

In particular, the function

$$
b(\gamma, x):=-\log \left|\gamma^{\prime}(x)\right|=B_{x}\left(\gamma^{-1} \cdot \mathbf{o}, \mathbf{o}\right)
$$

satisfies the cocycle relation $b\left(\gamma_{1} \gamma_{2}, x\right)=b\left(\gamma_{1}, \gamma_{2} \cdot x\right)+b\left(\gamma_{2}, x\right)$

Notation. - If $E$ is a subset of the boundary, $E \stackrel{\Delta}{\times} E$ denotes the complement of the diagonal in $E \times E$.

For a unit vector $v$ in the unit tangent bundle of the space $X$, let $v_{t}$ be the point at distance $t$ on the geodesic starting at $v$. Hence, $v_{0}$ is the base point of $v$, whereas $v_{-\infty}$ and $v_{+\infty}$ are the endpoints on the boundary of the geodesic. Associating to a vector $v$ the triplet $\left(v_{-\infty}, v_{+\infty}, r\right)$ where $r=B_{v_{+\infty}}\left(v_{0}, \mathbf{o}\right)$ gives an identification of $T^{1} X$ with the set $\partial X \stackrel{\Delta}{\times} \partial X \times \mathbb{R}$. With these coordinates, the geodesic flow $\left(\widetilde{g}_{t}\right)$ of the hyperbolic space acts by

$$
\widetilde{g}_{t}(y, x, r)=(y, x, r-t) .
$$

The action of an isometry $\gamma$ on $T^{1} X$ in these coordinates is given by

$$
\gamma \cdot(y, x, r)=(\gamma \cdot y, \gamma \cdot x, r-b(\gamma, x)) .
$$

A Kleinian group $\Gamma$ is a discrete torsion free subgroup of $\operatorname{Iso}(X)$. The limit set $\Lambda(\Gamma)$ is the smallest $\Gamma$-invariant closed subset of $\bar{X}=X \cup \partial X$. It is also the closure in the boundary of the set of fixed points of $\Gamma-\{\operatorname{Id}\}$ and can be obtained as the set of accumulation points in $\bar{X}$ of any $\Gamma$-orbit. A point $x$ in $\Lambda(\Gamma)$ is called radial if the geodesic ray $[\mathbf{o} x]$ stays at a bounded distance of the orbit $\Gamma \cdot \mathbf{o}$. For instance, fixed points of hyperbolic isometries of $\Gamma$ belong 
to the radial limit set whereas parabolic points, i.e. fixed points of parabolic isometries of $\Gamma$, do not. A parabolic point $x \in \Lambda(\Gamma)$ is said to be bounded if its stabilizer in $\Gamma$ acts on $\Lambda(\Gamma)-\{x\}$ with compact fundamental domain.

A conformal measure of dimension $\alpha \geq 0$ for $\Gamma$ is a family $\sigma=\left(\sigma_{\mathbf{x}}\right)_{\mathbf{x} \in X}$ of finite measures supported on the limit set of $\Gamma$ and such that, for any $\mathbf{x}, \mathbf{x}^{\prime} \in X$ and any $\gamma \in \Gamma$, one has

$$
\frac{\mathrm{d}\left(\gamma^{*} \sigma_{\mathbf{x}^{\prime}}\right)}{\mathrm{d} \sigma_{\mathbf{x}}}(x)=\mathrm{e}^{-\alpha \mathcal{B}_{x}\left(\mathbf{x}^{\prime}, \mathbf{x}\right)} \quad \text { and } \quad \gamma^{*} \sigma_{\mathbf{x}}=\sigma_{\gamma \cdot \mathbf{x}}
$$

(where $\gamma^{*} \sigma(A)=\sigma(\gamma A)$ for any Borel subset of $\partial X$ ).

By a result of Patterson [29], there always exists a conformal measure of dimension $\delta$, where $\delta$ is the critical exponent of $\Gamma$, that is the critical exponent of the Poincaré series of $\Gamma$

$$
P_{\Gamma}(s)=\sum_{\gamma \in \Gamma} \mathrm{e}^{-s d(\mathbf{o}, \gamma \cdot \mathbf{o})} .
$$

In this paper, we shall mainly consider divergent Kleinian groups i.e. groups for which $P_{\Gamma}$ diverges at $s=\delta$. In this case, there exists a unique conformal measure for $\Gamma$ of dimension $\delta$. It gives full measure to the radial limit set and is called the Patterson measure of $\Gamma$.

Quotienting the space $X$ by $\Gamma$ leads to a negatively curved manifold $M=X / \Gamma$, whose unit tangent bundle is $T^{1} M=T^{1} X / \Gamma$. Since the geodesic flow $\left(\widetilde{g}_{t}\right)$ commutes with the action of $\Gamma$ on $T^{1} X^{n}$, it induces on $T^{1} M$ the geodesic flow $\left(g_{t}\right)$ of $M$. The non-wandering set $\Omega$ of $\left(g_{t}\right)$ identifies with the projection on $T^{1} M$ of the $\Gamma$-invariant subset $\widetilde{\Omega}:=\Lambda(\Gamma) \stackrel{\Delta}{\times} \Lambda(\Gamma) \times \mathbb{R}$ of $T^{1} X$ (see $[11])$.

As observed by Sullivan [36], the Patterson measure of $\Gamma$ can be used to construct an invariant measure for the geodesic flow with support $\Omega$. The measure $\widetilde{m}$ on $\widetilde{\Omega}$ given by

$$
\mathrm{d} \tilde{m}(y, x, t)=\frac{\mathrm{d} \sigma(y) \mathrm{d} \sigma(x)}{|y-x|^{2 \delta}} \mathrm{d} t
$$

is clearly $\left(\widetilde{g}_{t}\right)$ invariant, but it is also $\Gamma$-invariant according to the "mean value relation" (see [36]):

$$
|\gamma \cdot y-\gamma \cdot x|^{2}=\left|\gamma^{\prime}(y)\right| \cdot\left|\gamma^{\prime}(x)\right| \cdot|y-x|^{2} .
$$

Hence, it induces on $\Omega$ an invariant measure $m$ that we call the PattersonSullivan measure.

II-b. Schottky products. - In this subsection, we introduce Schottky products and give their main properties. These Schottky products will be typical examples of groups satisfying the following property:

TOME $134-2006-\mathrm{N}^{\mathrm{O}} 1$ 
Property C. - There exist a closed and proper subset F in the boundary of the hyperbolic space such that any element of $\Gamma$ different from the identity maps $\partial X-F$ into $F$.

We now consider a group $\Gamma$ satisfying Property $C$. We have the two following facts:

FACT 1. - The group $\Gamma$ is discrete and its limit set is contained in $F$.

FACT 2. - For any compact set $K$ of the boundary and disjoint from $F$, there exists $C>0$ such that, for any $x \in K$ and $\gamma \in \Gamma$ one has

$$
d(\mathbf{o}, \gamma \cdot \mathbf{o})-C \leq B_{x}(\gamma \cdot \mathbf{o}, \mathbf{o}) \leq d(\mathbf{o}, \gamma \cdot \mathbf{o}) .
$$

Proof of Fact 1. - If $\Gamma$ was not discrete, one would get in $\Gamma$ a sequence of elements acting on $\partial X$ and converging to the identity so that any point outside $F$ would be the limit of a sequence of points in $F$. That the limit set of $\Gamma$ is contained in $F$ follows from the fact that fixed points of isometries in $\Gamma^{*}=\Gamma-\{\operatorname{Id}\}$ belong to $F$.

Proof of Fact 2. - To check this, recall that the orbit $\Gamma \cdot \mathbf{o}$ accumulates in $F$. Thus, if $\mathbf{x} \in X$ is close to $K$, the angle at $\mathbf{o}$ of the triangle $(\gamma \cdot \mathbf{o}, \mathbf{o}, \mathbf{x})$ is bounded away from 0; Fact 2 now follows from an argument of comparation of triangles and a classical fact in hyperbolic geometry.

We can now state the definition of a Schottky product:

DEFINITION II.1. - Let $\Gamma_{i}, i=1, \ldots L$, be a family of torsion-free subgroups of $\operatorname{Iso}(X)$. These groups are said to be in a Schottky position if there exist disjoint closed sets $F_{i}$ in $\partial X$ such that

$$
\Gamma_{i}^{*}\left(\partial X-F_{i}\right) \subset F_{i}
$$

The group $\Gamma$ generated by the groups $\Gamma_{i}$ is called the Schottky product of the $\Gamma_{i}$ 's and denoted $\Gamma=\Gamma_{1} * \Gamma_{2} * \cdots * \Gamma_{L}$ or else by $\Gamma=*_{i} \Gamma_{i}$.

Since any element in $\Gamma^{*}$ maps the complement of the closed set $F=\bigcup F_{i}$ into $F$, the group $\Gamma$ is discrete by Fact 1 . Moreover, by the Klein's tennis table criteria, it is the free product of the groups $\Gamma_{i}$ : any element in $\Gamma$ can be uniquely written as the product

$$
\gamma=a_{1} \cdots a_{k}
$$

for some $a_{j} \in \bigcup \Gamma_{i}^{*}$ with the property that no two consecutive elements $a_{j}$ belong to the same group. The set $\mathcal{A}=\bigcup \Gamma_{i}^{*}$ is called the alphabet of $\Gamma$, and $a_{1}, \ldots, a_{k}$ the letters of $\gamma$. The number $k$ of letters is the symbolic length of $\gamma$. The last letter of $\gamma$ will play a special role, and the index of the group it belongs to will be denoted by $i(\gamma)$. Applying Fact 2 one gets

BULletin DE LA SOCiÉtÉ MATHÉMATIQUE DE FRANCE 
FACT 3. - There exists a constant $C>0$ such that

$$
d(\mathbf{o}, \gamma \cdot \mathbf{o})-C \leq B_{x}\left(\gamma^{-1} \cdot \mathbf{o}, \mathbf{o}\right) \leq d(\mathbf{o}, \gamma \cdot \mathbf{o})
$$

for any $\gamma \in \Gamma=*_{i} \Gamma_{i}$ and any $x \in \bigcup_{i \neq i(\gamma)} F_{i}$.

This fact implies in particular the following crucial contraction property:

Proposition II.2. - There exist a real number $r \in] 0,1[$ and $C>0$ such that for any $\gamma$ with symbolic length $n \geq 1$ and any $x$ belonging to the closed set $\bigcup_{i \neq i(\gamma)} F_{i}$ one has

$$
\left|\gamma^{\prime}(x)\right| \leq C r^{n}
$$

Proof. - Recall that the conformal factor of $\gamma$ is $\left|\gamma^{\prime}(x)\right|=\mathrm{e}^{-B_{x}\left(\gamma^{-1} \mathbf{o}, \mathbf{o}\right)}$. By Fact 3 and the discreteness of $\Gamma$, we get $\left|\gamma^{\prime}(x)\right| \leq \frac{1}{2}$ for all but finitely many $\gamma \in \Gamma$ and all $x \in \bigcup_{i \neq i(\gamma)} F_{i}$; this inequality holds in particular for any isometry $\gamma$ of symbolic length $\geq N$ where $N$ is large enough. The proposition follows with $C=\sup \left\{\left|\gamma^{\prime}(x)\right| ; \gamma \in \Gamma, x \in \bigcup_{i \neq i(\gamma)} F_{i}\right\}$ and $r=1 / \sqrt[N]{2}$.

II-c. The limit set of $\Gamma=*_{i} \Gamma_{i}$ and the Patterson measure. - Let $\Gamma$ be a Schottky product as above. The following proposition gives a description of a large part of its limit set $\Lambda(\Gamma)$ (see [30]).

Proposition II.3. - Denote by $\Sigma^{+}$the set of sequences $\left(a_{n}\right)_{n \geq 0}$ for which each letter $a_{n}$ belongs to the alphabet $\mathcal{A}=\bigcup \Gamma_{i}^{*}$ and such that no two consecutive letters belong to the same group (these sequences are called admissible). Fix a point $x_{0}$ in $\partial X-F$. Then

a) For any $\mathbf{a}=\left(a_{n}\right) \in \Sigma^{+}$, the sequence $a_{0} \cdots a_{n} \cdot x_{0}$ converges to a point $\pi(\mathbf{a})$ in the limit set of $\Gamma$, independent on the choice of $x_{0}$.

b) The map $\pi: \Sigma^{+} \rightarrow \Lambda(\Gamma)$ is one-to-one and $\pi\left(\Sigma^{+}\right)$is contained in the radial limit set of $\Gamma$.

c) The complement of $\pi\left(\Sigma^{+}\right)$in the limit set of $\Gamma$ consists of the $\Gamma$-orbit of the union of the limits sets $\Lambda\left(\Gamma_{i}\right)$

The limit sets $\Lambda\left(\Gamma_{i}\right)$ are proper subsets of $\Lambda(\Gamma)$; furthermore the critical exponent $\delta_{i}$ of each subgroup $\Gamma_{i}, 1 \leq i \leq L$, is less or equal to the one of $\Gamma$ since the $\Gamma_{i}$ are subgroups of $\Gamma$. Recall that $\Gamma$ satisfies the critical gap property when $\delta>\delta_{\Gamma_{i}}$ for any $i \in\{1, \ldots, L\}$. One has the

TheOrem II.4. - (See [30]) Let $\Gamma_{i}, i=1, \ldots L$ be a finite collection of Kleinian groups in a Schottky position. Then if $\Gamma_{i}$ is divergent for any $i=1, \ldots, L$, the group $\Gamma=*_{i} \Gamma_{i}$ is also divergent. Moreover

- $\delta>\max _{i} \delta_{\Gamma_{i}}$;

- the Patterson measure of $\Gamma$ gives measure 0 to the limit sets $\Lambda\left(\Gamma_{i}\right)$;

- the Patterson-Sullivan measure is finite.

TOME $134-2006-\mathrm{N}^{\mathrm{O}} 1$ 


\section{REMARKS.}

a) If the groups $\Gamma_{i}$ are elementary Kleinian groups, their limit sets is reduced to one point or two points, and the above result shows that $\sigma$ has no atoms.

b) The conclusions of this theorem still hold when one only assume that one of the groups $\Gamma_{i}$ of maximal exponent is divergent (see [30]).

c) If the groups $\Gamma_{i}$ are geometrically finite, it can be checked that the product is also geometrically finite (see [26]).

\section{Symbolic dynamic and stochastic behavior of the geodesic flow}

From now on, we consider a Schottky product group $\Gamma$. Thus, up to a set of measure 0 , the limit set of $\Gamma$ coincides with $\Lambda:=\pi\left(\Sigma^{+}\right)$. Let $\Lambda_{i}$ be the subset of $\Lambda$ of those limit points with first letter in $\Gamma_{i}$ (not to be confused with the limit set of $\Gamma_{i}$ ). The following description of $\Lambda$ will be useful:

a) $\Lambda$ is the finite union of the sets $\Lambda_{i}$, with disjoint closures $\Lambda(\Gamma) \cap F_{i}$.

b) Each of these sets is partitioned into a countable number of subsets with disjoint closures:

$$
\Lambda_{i}=\bigcup_{a \in \Gamma_{i}^{*}} \bigcup_{j \neq i} a \cdot \Lambda_{j} .
$$

III-a. Coding the geodesic flow. - In this set-up, the non-wandering set $\Omega$ of the geodesic flow contains $\Omega^{\prime}=\Lambda \times \Delta \times \times \mathbb{R} / \Gamma$ as a subset of full measure with respect to the Patterson-Sullivan measure, and we can therefore restrict $\left(g_{t}\right)$ to $\Omega^{\prime}$. We shall now produce a coding of the geodesic flow on $\Omega^{\prime}$ by first conjugating the action of $\Gamma$ on $\Lambda \times \Delta \times \mathbb{R}$ with the action of a single transformation. Observe that the subset $\bar{\Lambda}=\bigcup_{i \neq j} \Lambda_{i} \times \Lambda_{j}$ of $\Lambda \times \Delta$ is in a one-to-one correspondence with the symbolic space $\Sigma$ of bi-infinite admissible sequences in $\mathcal{A}^{\mathbb{Z}}$, and that the shift of $\Sigma$ induces a transformation $\bar{T}$ on this set $\bar{\Lambda}$ given by

$$
\bar{T}(y, x)=\left(a_{0}^{-1} y, a_{0}^{-1} x\right) \quad \text { if } \quad x=\pi(\mathbf{a})
$$

Lemma III.1. - The action of $\Gamma$ on $\Lambda \stackrel{\Delta}{\times} \Lambda$ is orbit equivalent with the action of $\bar{T}$ on $\bar{\Lambda}$.

Proof. - Let $x=\pi(\mathbf{a})$ and $y=\pi(\mathbf{b})$ two distinct points in $\Lambda$, and denote by $n$ the first index for which $a_{n} \neq b_{n}$. Let $\gamma=a_{0} \cdots a_{n-1}=b_{0} \cdots b_{n-1}$. Then, $\gamma^{-1}(y, x)$ belongs to $\bar{\Lambda}$. Therefore, the $\Gamma$-orbit of $(y, x)$ meets $\bar{\Lambda}$ which means that $\bar{\Lambda}$ is a section for the action of $\Gamma$. Furthermore, when $(y, x)$ belongs to $\pi(\Sigma)$, it is easily checked that $\gamma(y, x) \in \bar{\Lambda}$ if and only if $\gamma=\left(a_{0} \cdots a_{k-1}\right)^{-1}$ or $\gamma=b_{0} \cdots b_{k-1}$ for some $k \geq 0$. In the first case, $\gamma(y, x)=\bar{T}^{k}(y, x)$, and in the second case, $\gamma(y, x)=\bar{T}^{-k}(y, x)$, which proves the lemma. 
It is now a consequence of Lemma III.1 that the action of $\Gamma$ on the space $\Lambda \stackrel{\Delta}{\times} \Lambda \times \mathbb{R}$ is orbit-equivalent with the transformation $\bar{T}_{\ell}$ on $\bar{\Lambda} \times \mathbb{R}$ given by

$$
\bar{T}_{\ell}(y, x, r)=a_{0}^{-1}(y, x, r)=(\bar{T}(y, x), r+\ell(x))
$$

where, according to (1), the function $\ell$ is defined by

$$
\ell(x)=-b\left(a_{0}^{-1}, x\right)=-B_{x}\left(a_{0} \mathbf{o}, \mathbf{o}\right) \quad \text { when } \quad x=\pi(\mathbf{a})
$$

It follows that the geodesic flow $\left(g_{t}\right)$ of $M$ (restricted to $\Omega^{\prime}$ ) can be presented as the special flow on the space $\bar{\Lambda} \times \mathbb{R} / \bar{T}_{\ell}$. When the function $\ell$ is non negative on $\Lambda$, the set

$$
D_{\ell}=\{(y, x, s) ;(y, x) \in \bar{\Lambda}, s \in[0, \ell(x)[\}
$$

is a fundamental domain for the action of $\bar{T}_{\ell}$ on $\bar{\Lambda} \times \mathbb{R}$. In the general case one only knows by Fact 3 in the previous section that $\ell$ is bounded from below and $S_{N} \ell=\ell+\ell \circ T+\cdots+\ell \circ T^{N-1}$ is non negative on $\Lambda$ for some $N \geq 1$; the function $\ell$ is thus cohomologous to a strictly positive function $\ell^{+}$(e.g. $\ell=\ell^{+}+h-h \circ T$ for some measurable function $h$ ) and the set $\left\{(y, x, s) ;(y, x) \in \bar{\Lambda}, h(x) \leq s<h(x)+\ell^{+}(x)\right\}$ is a fundamental domain for the action of $\bar{T}_{\ell}$ on $\bar{\Lambda} \times \mathbb{R}$.

In order to simplify the notations, we will assume in the sequel that $\ell$ is non negative on $\Lambda$ and identifies $\bar{\Lambda} \times \mathbb{R} / \bar{T}_{\ell}$ with $D_{\ell}$ defined above.

Note that restricting the measure $\mathrm{d} \sigma(y) \mathrm{d} \sigma(x) /|y-x|^{2 \delta}$ to $\bar{\Lambda}$ and normalising it gives an invariant probability measure for $\bar{T}$, that we shall denote by $\bar{\nu}$. The measure $\bar{\nu}(\mathrm{d} y \mathrm{~d} x) \mathrm{d} t$ on $\bar{\Lambda} \times \mathbb{R}$ is thus $\bar{T}_{\ell}$-invariant, it induces an invariant measure for the special flow on the space $\bar{\Lambda} \times \mathbb{R} / \bar{T}_{\ell}$ which corresponds with the Patterson-Sullivan measure on $\Omega$.

III-b. The factor $(\boldsymbol{\Lambda}, \boldsymbol{T}, \boldsymbol{\nu})$. - We have constructed a dynamical system $(\bar{\Lambda}, \bar{T}, \bar{\nu})$, isomorphic to a symbolic space of bi-infinite sequences such that the geodesic flow restricted to $\Omega^{\prime}$ is a suspension of it. By using the projection $p: \bar{\Lambda} \rightarrow \Lambda$ on the second coordinate, we get a factor $(\Lambda, T, \nu)$ of the dynamical system $(\bar{\Lambda}, \bar{T}, \bar{\nu})$ that we now describe.

The transformation $T$ is induced by the unilateral shift and is simply given by

$$
T x=a_{0}^{-1} \cdot x \quad \text { if } \quad x=\pi(\mathbf{a}) .
$$

This transformation is uniformly expanding by Proposition II.2.

The probability measure $\nu=\bar{\nu} \circ p^{-1}$ on $\Lambda$ is invariant for $T$ and has a density $h$ with respect to the Patterson measure $\sigma$ on $\Lambda$ given up to a normalization constant by the formula

$$
\text { for } x \in \Lambda_{i}, \quad h(x)=\int_{\bigcup_{j \neq i} \Lambda_{j}} \frac{\mathrm{d} \sigma(y)}{|x-y|^{2 \delta}} .
$$

TOME $134-2006-\mathrm{N}^{\mathrm{O}} 1$ 
III-c. The transfer operator. - Classically, the stochastic behavior of a special flow over a subshift of finite type $(\Sigma, \sigma)$ with ceiling function $\ell$ is studied using the factor system $\left(\Sigma^{+}, \sigma^{+}\right)$of one-sided sequences and the family of transfer operators $\left(\mathcal{L}_{z}\right)_{z}$ defined by

$$
\mathcal{L}_{z} \varphi(x)=\sum_{\sigma y=x} \mathrm{e}^{-z \ell(y)} \varphi(y) .
$$

In this section, we study the family of transfer operators associated with the dynamical system $(\Lambda, T, \nu)$ and the ceiling function $\ell$ defined above, taking into account that our alphabet is countable; these operators $\mathcal{L}_{z}$ will be defined formally by

$$
\mathcal{L}_{z} \varphi(x)=\sum_{T y=x} \mathrm{e}^{-z f(y)} \varphi(y) .
$$

for any function $\varphi$ from $\Lambda$ to $\mathbb{C}$.

If $x$ belongs to $\Lambda_{i}$, its pre-images by $T$ are the points $y=a \cdot x$ for $a \in \bigcup_{j \neq i} \Gamma_{j}^{*}$ and then $\ell(y)=-b\left(a^{-1}, a \cdot x\right)=b(a, x)$; one may then set

$$
\mathcal{L}_{z} \varphi(x)=\sum_{i \neq j} \sum_{a \in \Gamma_{j}^{*}} 1_{x \in \Lambda_{i}} \mathrm{e}^{-z b(a, x)} \varphi(a x) .
$$

if $x \in \Lambda_{i}$. Assume that $\varphi$ is bounded. By Fact 3, the series above converges as soon as $\Re(z)$ is strictly greater than the critical exponent of each group $\Gamma_{i}$, $1 \leq i \leq \ell$; this occurs in particular if $z=\delta$ in our set-up by the critical gap property given in Theorem II.4.

According to the next proposition, the operator $\mathcal{L}_{\delta}$ can be seen as a dual operator of the transformation $T$ acting on $L^{\infty}(\Lambda(\Gamma))$ endowed with the Patterson measure $\sigma$.

Proposition III.2. - For any $\phi$ and $\psi$ in $L^{\infty}(\Lambda(\Gamma))$, one has

$$
\int_{\Lambda} \varphi(x) \psi(T x) \sigma(\mathrm{d} x)=\int_{\Lambda} \mathcal{L}_{\delta} \varphi(x) \psi(x) \sigma(\mathrm{d} x) .
$$

In particular, the Patterson measure $\sigma$ is an invariant measure for $\mathcal{L}_{\delta}$ and the density $h$ defined in Section III-b is an invariant function:

$$
\sigma \mathcal{L}_{\delta}=\sigma \quad \text { and } \quad \mathcal{L}_{\delta} h=h .
$$

Proof. - Recall from Section 3 that $\Lambda$ coincides with $\Lambda(\Gamma)$ up to a subset of Patterson's measure 0 and can be partitioned into a countable number of subsets with disjoint closures

$$
\Lambda=\bigcup_{i \neq j} \bigcup_{a \in \Gamma_{j}^{*}} a \Lambda_{i} .
$$

BULlETiN DE LA SOCIÉtÉ MATHÉMATIQUE DE FRANCE 
The restriction of the transformation $T$ on $a \Lambda_{i}$ is the action of $a^{-1}$; the $\delta$ conformality of the Patterson's measure $\sigma$ thus implies that, for any $\varphi, \psi$ in $L^{\infty}(\Lambda(\Gamma))$,

$$
\begin{aligned}
\int_{\Lambda(\Gamma)} \varphi(x) \psi(T x) \sigma(\mathrm{d} x) & =\sum_{i \neq j} \sum_{a \in \Gamma_{j}^{*}} \int_{a \Lambda_{i}} \varphi(x) \psi(T x) \sigma(\mathrm{d} x) \\
& =\sum_{i \neq j} \sum_{a \in \Gamma_{j}^{*}} \int_{\Lambda_{i}} \varphi(a \cdot x) \psi(x)\left|a^{\prime}(x)\right|^{\delta} \sigma(\mathrm{d} x) .
\end{aligned}
$$

Recalling that $\left|a^{\prime}(x)\right|=\mathrm{e}^{-b(a, x)}$, we get $(3)$. For $\psi=1_{\Lambda(\Gamma)}$ one obtains in particular $\sigma \mathcal{L}_{\delta}=\sigma$. Furthermore since $\nu=h \sigma$ is $T$-invariant one gets $\mathcal{L}_{\delta} h=h$.

In order to control more precisely the spectrum of the transfer operators, we will study their restriction the space $L_{\alpha}=L_{\alpha}(\Lambda(\Gamma))$ of Hölder continuous functions from $\Lambda(\Gamma)$ to $\mathbb{C}$ defined by

$$
L_{\alpha}=\left\{\varphi \in C(\Lambda(\Gamma)) ;\|\varphi\|_{\alpha}=|\varphi|_{\infty}+[\varphi]_{\alpha}<+\infty\right\}
$$

where $[\varphi]_{\alpha}$ is the $\alpha$-Hölder coefficient of $\varphi$ defined by

$$
[\varphi]_{\alpha}=\sup _{i} \sup _{\substack{x, y \in \bar{\Lambda}_{i} \\ x \neq y}} \frac{|\varphi(x)-\varphi(y)|}{|x-y|^{\alpha}}
$$

(the set $\bar{\Lambda}_{i}$ denotes here the set $\Lambda(\Gamma) \cap F_{i}$, that is the closure of $\Lambda_{i}$ ). The space $\left(L_{\alpha},\|.\|_{\alpha}\right)$ is a Banach space and by Ascoli's theorem the identity map from $\left(L_{\alpha},\|\cdot\|_{\alpha}\right)$ into $\left(C(\Lambda(\Gamma)),|\cdot|_{\infty}\right)$ is compact.

For any $z \in \mathbb{C}$ and $\gamma$ in $\Gamma^{*}$, let $w_{z}(\gamma,$.$) be the weight function defined on$ $\Lambda(\Gamma)$ by

$$
w_{z}(\gamma, x)= \begin{cases}0 & \text { if } x \in \bar{\Lambda}_{i(\gamma)} \\ \mathrm{e}^{-z b(\gamma, x)} & \text { if } x \in \bar{\Lambda}_{i}, i \neq i(\gamma) .\end{cases}
$$

Observe that these weights satisfy the following cocycle relation: if $a_{1}, a_{2} \in \mathcal{A}$ do not belong to the same group $\Gamma_{i}$, then

$$
w_{z}\left(a_{1} a_{2}, x\right)=w_{z}\left(a_{1}, a_{2} \cdot x\right) w_{z}\left(a_{2}, x\right) .
$$

LEMMA III.3. - Each weight $w_{z}(\gamma,$.$) belongs to L_{\alpha}$ for any $0<\alpha \leq 1$. Moreover, for any $z \in \mathbb{C}$ there exists $C=C(z)>0$ such that for any $\gamma$ in $\Gamma^{*}$,

$$
\left\|w_{z}(\gamma, .)\right\|_{\alpha} \leq C \mathrm{e}^{-\Re(z) d(\mathbf{o}, \gamma \cdot \mathbf{o})}
$$

Proof. - Fact 3 implies that the family $\left\{\mathrm{e}^{\Re(z) d(\mathbf{o}, \gamma \cdot \mathbf{o})} w_{z}(\gamma,.) ; \gamma \in \Gamma\right\}$ is bounded for $|\cdot|_{\infty}$. The control of the Lipschitz-coefficient of $w_{z}(\gamma,$.$) is more$ delicate.

TOME $134-2006-\mathrm{N}^{\mathrm{O}} 1$ 
We first recall briefly the proof in the constant curvature case. To estimate $w_{z}(\gamma, x)-w_{z}(\gamma, y)$ for any points $x, y$ belonging to the same subset $\Lambda_{i}$ with $i \neq i(\gamma)$, note that there exists a constant $A>0$ such that $|b(\gamma, x)-b(\gamma, y)| \leq A|x-y|$. The inequality $\left|\mathrm{e}^{Z}-1\right| \leq 2|Z| \mathrm{e}^{|\Re(Z)|}$ readily implies

$$
\left|\mathrm{e}^{-z b(\gamma, x)}-\mathrm{e}^{-z b(\gamma, y)}\right| \leq A|z| \mathrm{e}^{2 A|\Re(z)|-\Re(z) b(\gamma, x)}|x-y| .
$$

So, the Hölder coefficient of $w_{z}(\gamma,$.$) satisfies \left[w_{z}(\gamma, .)\right]_{\alpha} \leq C \mathrm{e}^{-\Re(z) d(\mathbf{o}, \gamma \cdot \mathbf{o})}$ for some constant $C=C(z)$.

In the variable curvature case, we same argument holds using the following fact which extends a result due to M. Bourdon [4].

FACT 4. - Let $E \subset \partial X$ and $F \subset X$ two sets whose closure $\bar{E}$ and $\bar{F}$ in $X \cup \partial X$ are disjoint. Then the functions $x \mapsto \mathcal{B}_{x}(\mathbf{o}, \mathbf{p})$, with $\mathbf{p} \in F$, are equi-Lipschitz continuous on $E$ with respect to |.|.

Proof. - For any points $\mathbf{p} \in X$ and $x, y \in \partial X$, the quantity

$$
(x \mid y)_{\mathbf{p}}=\frac{1}{2}\left(\mathcal{B}_{x}(\mathbf{p}, \mathbf{q})+\mathcal{B}_{y}(\mathbf{p}, \mathbf{q})\right)
$$

does not depend on the point $\mathbf{q}$ on the geodesic $(x y)$; furthermore the function $\Delta_{\mathbf{p}}$ defined by $\Delta_{\mathbf{p}}(x, x)=0$ and $\Delta_{\mathbf{p}}(x, y)=\exp \left(-(x \mid y)_{\mathbf{p}}\right)$ for $x \neq y$ is a distance on $\partial X$ which satisfies the well known visibility property:

There exists $C>0$, such that, for any couple $(x, y)$ of distinc points in $\partial X$

$$
C^{-1} \exp \left(-d(\mathbf{p},(x y)) \leq \Delta_{\mathbf{p}}(x, y) \leq C \exp (-d(\mathbf{p},(x y)) .\right.
$$

Note that the distance $\Delta_{\mathbf{o}}(x, y)$ is nothing else than the distance $|x-y|$ introduced above; furthermore, the constant $C$ is a universal constant which depends only on the dimension of $X$ and the upper bound on the curvature (see [16]). The distances $\Delta_{\mathbf{p}}, \mathbf{p} \in X$, are equivalent on $\partial X$ since $\Delta_{\mathbf{p}}(x, y)=\Delta_{\mathbf{o}}(x, y) \exp \frac{1}{2}\left(\mathcal{B}_{x}(\mathbf{o}, \mathbf{p})+\mathcal{B}_{y}(\mathbf{o}, \mathbf{p})\right)$. This readily implies

$$
\mathcal{B}_{x}(\mathbf{o}, \mathbf{p})=-2 \log \Delta_{\mathbf{o}}(x, y)+2 \log \Delta_{\mathbf{p}}(x, y)-\mathcal{B}_{y}(\mathbf{o}, \mathbf{p}) .
$$

We will use the two following properties, whose proof is developed a bit latter:

i) There exists $\epsilon>0$ such that for any $\mathbf{p} \in F$ one can choose $x_{\mathbf{p}} \in \partial X$ such that $\Delta_{\mathbf{o}}\left(x_{\mathbf{p}}, E\right) \geq \epsilon$ and $\Delta_{\mathbf{p}}\left(x_{\mathbf{p}}, E\right) \geq \epsilon$.

ii) The set $\left\{d\left(\mathbf{p},\left(x x^{\prime}\right)\right)-d\left(\mathbf{o},\left(x x^{\prime}\right)\right)\right\}$ is bounded from below, uniformly in $\mathbf{p} \in F$ and $x, x^{\prime} \in E, x \neq x^{\prime}$.

Fix $\mathbf{p}$ in $F$. The quantity $\mathcal{B}_{x_{\mathbf{p}}}(\mathbf{o}, \mathbf{p})$ does not depend on $x$. On the other hand, the function $x \mapsto \Delta_{\mathbf{o}}\left(x, x_{\mathbf{p}}\right)$ is Lipschitz continuous (with coefficient 1) with respect to $\Delta_{\mathbf{o}}$; since it is bounded from below by $\epsilon$, the function $x \mapsto$ $\log \Delta_{\mathbf{o}}\left(x, x_{\mathbf{p}}\right)$ is also Lipschitz continuous with respect to $\Delta_{\mathbf{o}}$ and its Lipschitz coefficient is uniformly bounded in $\mathbf{p}$. In the same way, the function 
$x \mapsto \log \Delta_{\mathbf{p}}\left(x, x_{\mathbf{p}}\right)$ is Lipschitz continuous with respect to $\Delta_{\mathbf{p}}$, with a Lipschitz coefficient uniformly bounded in $\mathbf{p}$. Fact 4 will be proved if one shows that $\Delta_{\mathbf{p}}\left(x, x^{\prime}\right) \leq C \Delta_{\mathbf{o}}\left(x, x^{\prime}\right)$ for some constant $C>0$ which does not depend on $p \in F$; this is the property ii).

It remains to prove assertions i) and ii).

Proof of $i$ ). - If $F$ is relatively compact in $X$, one fixes $x_{0}$ in $\partial X$ outside $\bar{E}$ and one set $x_{\mathbf{p}}=x_{0}$ for any $\mathbf{p} \in F$. In the sequel we will assume that $F$ is not bounded in $X$, we set $\epsilon_{0}=\frac{1}{2} \Delta_{\mathbf{o}}(E, F \cap \partial X)>0$ and we choose a compact subset $K_{0}$ of $\partial X$ such that, if $x, y$ are two distinct points in $\partial X$ with $\Delta_{\mathbf{o}}(x, y) \geq \epsilon_{0}$ then the geodesic $(x y)$ meets the set $K_{0}$. Fix $e_{0} \in E$ and $x_{0} \in \partial X$ with $\Delta_{\mathbf{o}}\left(x_{0}, E\right) \geq \epsilon_{0}$. When $\mathbf{p} \in F$ lies inside $K_{0}$ set $x_{\mathbf{p}}=x_{0}$; otherwise let $x_{\mathbf{p}}$ be the point in $\partial X$ such that the geodesic $\left(e_{0} x_{\mathbf{p}}\right)$ contains $\mathbf{p}$. One can enlarge $K_{0}$ in such a way that $\Delta_{\mathbf{o}}\left(x_{\mathbf{p}}, E\right) \geq \epsilon_{0}$ as soon as $\mathbf{p} \notin K_{0}$.

- If $\mathbf{p} \in K_{0}$, one has $x_{\mathbf{p}}=x_{0}$ and so $\Delta_{\mathbf{o}}\left(x_{\mathbf{p}}, E\right) \geq \epsilon_{0}$; it follows that, for any $e \in E$, the geodesic $\left(e x_{\mathbf{p}}\right)$ cuts the set $K_{0}$ and so $d\left(\mathbf{p},\left(e x_{\mathbf{p}}\right)\right) \leq \operatorname{diam}\left(K_{0}\right)$.

- If $\mathbf{p} \notin K_{0}$, one has $\Delta_{\mathbf{o}}\left(x_{\mathbf{p}}, E\right) \geq \epsilon_{0}$ and for any $e \in E$, one can thus choose a point $\mathbf{q}_{e}$ on the geodesic $\left(x_{\mathbf{p}} e\right)$ which belongs to $K_{0}$. In particular the point $\mathbf{p}$ is on the geodesic ray $\left[\mathbf{q}_{e_{0}}, x_{\mathbf{p}}\right)$, which readily implies

$$
d\left(\mathbf{p},\left(e x_{\mathbf{p}}\right)\right) \leq d\left(\mathbf{q}_{e_{0}} \mathbf{q}_{e}\right) \leq 2 \operatorname{diam}\left(K_{0}\right) .
$$

In the two cases, one concludes with the visibility property.

Proof of $i i)$. - Assume that there exists sequences $\left(\mathbf{p}_{n}\right)$ in $F$ and $\left(x_{n}\right)$, $\left(x_{n}^{\prime}\right)$ in $E$, with $x_{n} \neq x_{n}^{\prime}$, such that $d\left(\mathbf{p}_{n},\left(x_{n} x_{n}^{\prime}\right)\right)-d\left(\mathbf{o},\left(x_{n} x_{n}^{\prime}\right)\right) \rightarrow-\infty$ as $n \rightarrow+\infty$. Let $\mathbf{q}_{n}$ be the point on the geodesic $\left(x_{n} x_{n}^{\prime}\right)$ such that $d\left(\mathbf{p}_{n},\left(x_{n} x_{n}^{\prime}\right)\right)=$ $d\left(\mathbf{p}_{n}, \mathbf{q}_{n}\right)$; one has $d\left(\mathbf{p}_{n}, \mathbf{q}_{n}\right)-d\left(\mathbf{o}, \mathbf{q}_{n}\right) \rightarrow-\infty$. Taking if necessary a subsequence, one may assume that $\left(\mathbf{p}_{n}\right)_{n}$ and $\left(\mathbf{q}_{n}\right)_{n}$ converge respectively to some points $p$ and $q$. Since $d\left(\mathbf{p}_{n}, \mathbf{q}_{n}\right)-d\left(\mathbf{o}, \mathbf{q}_{n}\right) \rightarrow-\infty$, one has $d\left(\mathbf{o}, \mathbf{q}_{n}\right) \rightarrow+\infty$ and so $p \in E$; one also has $d\left(\mathbf{o}, \mathbf{p}_{n}\right) \rightarrow+\infty$ because $\left|d\left(\mathbf{p}_{n}, \mathbf{q}_{n}\right)-d\left(\mathbf{o}, \mathbf{q}_{n}\right)\right| \leq d\left(\mathbf{o}, \mathbf{p}_{n}\right)$, which implies that $q \in F \cap \partial X$. The angle at $\mathbf{o}$ in the triangle $(\mathbf{o} p q)$ is bounded from below by $\theta_{0}>0$; for $n$ large enough the angle between the two geodesic segments $\left[\mathbf{o}, \mathbf{p}_{n}\right]$ and $\left[\mathbf{o}, \mathbf{q}_{n}\right]$ is thus $\geq \frac{1}{2} \theta_{0}$ which implies that $d\left(\mathbf{p}_{n}, \mathbf{q}_{n}\right)-d\left(\mathbf{o}, \mathbf{p}_{n}\right)-d\left(\mathbf{o}, \mathbf{q}_{n}\right)$ is bounded from below. This contradicts the fact that $d\left(\mathbf{p}_{n}, \mathbf{q}_{n}\right)-d\left(\mathbf{o}, \mathbf{q}_{n}\right)$ tends to $-\infty$.

Lemma III.3 thus proves that $\mathcal{L}_{z}$ is bounded on $L_{\alpha}$ when $\Re(z)>\delta_{i}$ for all $1 \leq i \leq L$ and in particular for $z=\delta$ by the critical gap property. The following proposition gives a precise description of the spectrum of $\mathcal{L}_{\delta}$ on $L_{\alpha}$.

Proposition III.4. - For any $0<\alpha \leq 1$, the spectral radius of $\mathcal{L}_{\delta}$ on $L_{\alpha}$ is equal to 1 . Furthermore

- if $L \geq 3$, the eigenvalue 1 is simple and the rest of the spectrum of $\mathcal{L}_{\delta}$ is contained in a disc of radius strictly less than 1 ;

TOME $134-2006-\mathrm{N}^{\mathrm{O}} 1$ 
- if $L=2$ (i.e. if $\left.\Gamma=\Gamma_{1} * \Gamma_{2}\right)$, the function $h\left(1_{\bar{\Lambda}_{1}}-1_{\bar{\Lambda}_{2}}\right)$ is an eigenfunction of $\mathcal{L}_{\delta}$ for the value -1 , the eigenvalues 1 and -1 are simple and the rest of the spectrum of $\mathcal{L}_{\delta}$ is contained in a disc of radius strictly less than 1.

Proof. - The study of the spectrum of $\mathcal{L}_{z}$ was already done in [9] and [2] when the Schottky factors of $\Gamma$ are elementary subgroups of Iso $\left(\mathbb{H}^{n}\right)$. The proof in this more general case is similar, and we recall it for the sake of completeness. It is decomposed into four steps.

Step 1. The essential spectral radius of $\mathcal{L}_{z}$. - We first obtain a contraction property for the iterated operators $\mathcal{L}_{z}^{k}$. Due to the cocycle property (4) of the weights, we may write

$$
\mathcal{L}_{z}^{k} \varphi(x)=\sum_{\gamma \in \Gamma(k)} w_{z}(\gamma, x) \varphi(\gamma \cdot x)
$$

where $\Gamma(k)$ is the set of elements of symbolic length $k$ in $\Gamma$. Therefore

$$
\begin{aligned}
\left|\mathcal{L}_{z}^{k} \varphi(x)-\mathcal{L}_{z}^{k} \varphi(y)\right| \leq \sum_{\gamma \in \Gamma(k)}\left|w_{z}(\gamma, x)\right| \cdot|\varphi(\gamma \cdot x)-\varphi(\gamma \cdot y)| & +\sum_{\gamma \in \Gamma(k)}\left[w_{z}(\gamma, .)\right]_{\alpha} \cdot|\varphi|_{\infty} \cdot|x-y|^{\alpha} .
\end{aligned}
$$

By Proposition II.2 and the mean value relation (2), there exist $C>0$ and $r<1$ such that $|\gamma \cdot x-\gamma \cdot y| \leq C r^{k}|x-y|$ whenever $x, y \in \Lambda_{i}, i \neq i(\gamma)$. This leads to the inequality

$$
\left[\mathcal{L}_{z}^{k} \varphi\right]_{\alpha} \leq r_{k}[\varphi]_{\alpha}+R_{k}|\varphi|_{\infty}
$$

where $r_{k}=\left(C r^{k}\right)^{\alpha}\left|\mathcal{L}_{\Re(z)}^{k} 1\right|_{\infty}$ and $R_{k}=\sum_{\gamma \in \Gamma(k)}\left[w_{z}(\gamma, .)\right]_{\alpha}$. Observe that

$$
\limsup _{k} r_{k}^{1 / k}=r^{\alpha} \limsup _{k}\left|\mathcal{L}_{\Re(z)}^{k} 1\right|_{\infty}^{1 / k}=r^{\alpha} \rho_{\infty}(\Re(z))
$$

where $\rho_{\infty}(\Re(z))$ is the spectral radius of the positive operator $\mathcal{L}_{\Re(z)}$ on $C(\Lambda(\Gamma))$.

Inequality (5) is crucial in the Ionescu-Tulcea-Marinescu theorem for quasicompact operators and appeared first in [10]. By Hennion's work [20], it implies that the essential spectral radius of $\mathcal{L}_{z}$ on $L_{\alpha}$ is less than $r^{\alpha} \rho_{\infty}(\Re(z))$; in other words any spectral value of $\mathcal{L}_{z}$ with modulus strictly larger than $r^{\alpha} \rho_{\infty}(\Re(z))$ is an eigenvalue with finite multiplicity and isolated in the spectrum of $\mathcal{L}_{z}$.

It follows in particular that the spectral radius of $\mathcal{L}_{z}$ on $L_{\alpha}$ is less than $\rho_{\infty}(\Re(z))$. Otherwise there would exist a spectral value $\lambda$ of modulus bigger than $\rho_{\infty}(\Re(z))$ which would be an eigenvalue by the previous result; if $\varphi \in L_{\alpha}$ was a corresponding eigenfunction, one would thus obtain $|\lambda| \cdot|\varphi| \leq \mathcal{L}_{\Re(z)}|\varphi|$ and so $|\lambda| \leq \rho_{\infty}(\Re(z))$, a contradiction.

Step 2. The peripherical spectrum of $\mathcal{L}_{\delta}$. - Recall from Proposition III.2 that $\mathcal{L}_{\delta} h=h$, and that $h$ belongs to $C(\Lambda(\Gamma))$. Since $h$ is strictly greater than 0 on $\Lambda(\Gamma)$, the spectral radius on $C(\Lambda(\Gamma))$ of the positive operator $\mathcal{L}_{\delta}$ is $\rho_{\infty}\left(\mathcal{L}_{\delta}\right)=\lim \sup _{+\infty}\left|\mathcal{L}_{\delta}^{k} 1\right|_{\infty}^{1 / k}=1$. 
By the first step it follows that the spectral radius of $\mathcal{L}_{\delta}$ in $L_{\alpha}$ is less that 1 , and since $h$ belongs to $L_{\alpha}$, is equal to 1 . Moreover, we know that, except for finitely many eigenvalues of modulus 1 , the spectrum of $\mathcal{L}_{\delta}$ is included in a disc of radius $<1$. We now describe the peripherical spectrum of $\mathcal{L}_{\delta}$.

Assume first that $L \geq 3$ and let us consider a function $\varphi \in L_{\alpha}$ such that $\mathcal{L}_{\delta} \varphi=\mathrm{e}^{i \theta} \varphi$ for some $\theta \in \mathbb{R}$. It follows that $|\varphi| \leq \mathcal{L}_{\delta}|\varphi|$ and so $|\varphi(x)|=$ $\mathcal{L}_{\delta}|\varphi|(x) \sigma(\mathrm{d} x)-$ a.s. since $\sigma \mathcal{L}_{\delta}=\sigma$. The equality holds in fact for all $x \in \Lambda(\Gamma)$ since $|\varphi|$ and $\mathcal{L}_{\delta}|\varphi|$ are continuous and the support of $\sigma$ is $\Lambda(\Gamma)$. Now, let $x_{0}$ in $\Lambda(\Gamma)$ such that $\left|\varphi\left(x_{0}\right)\right| / h\left(x_{0}\right)=\sup _{x \in \Lambda(\Gamma)}|\varphi(x)| / h(x)$ and denote by $i_{0}$ the index such that $x_{0}$ belongs to the closure of $\Lambda_{i_{0}}$; the equalities $\mathcal{L}_{\delta}|\varphi|=|\varphi|$ and $\mathcal{L}_{\delta} h=h$ imply by convexity

$$
\forall j \neq i_{0}, \forall a \in \Gamma_{j}^{*}, \quad \frac{\left|\varphi\left(a \cdot x_{0}\right)\right|}{h\left(a \cdot x_{0}\right)}=\frac{\left|\varphi\left(x_{0}\right)\right|}{h\left(x_{0}\right)} .
$$

In the same way, if $x_{1} \in \Lambda(\Gamma)$ satisfies $\left|\varphi\left(x_{1}\right)\right| / h\left(x_{1}\right)=\inf _{x \in \Lambda(\Gamma)}|\varphi(x)| / h(x)$ and if $x_{1}$ belongs to the closure of $\Lambda_{i_{1}}$, one has

$$
\forall j \neq i_{1}, \forall a \in \Gamma_{j}^{*}, \quad \frac{\left|\varphi\left(a \cdot x_{1}\right)\right|}{h\left(a \cdot x_{1}\right)}=\frac{\left|\varphi\left(x_{1}\right)\right|}{h\left(x_{1}\right)} .
$$

Since $L \geq 3$ there exists an index $j$ which is distinct from $i_{0}$ and $i_{1}$. Let $a \in \Gamma_{j}^{*}$; the sequences $\left(a^{n} \cdot x_{0}\right)_{n \geq 1}$ and $\left(a^{n} \cdot x_{1}\right)_{n \geq 1}$ converge to the attractive fixed point of $a$ and by continuity it follows that $\left|\varphi\left(x_{0}\right)\right| / h\left(x_{0}\right)=\left|\varphi\left(x_{1}\right)\right| / h\left(x_{1}\right)$; the function $|\varphi| / h$ is thus constant on $\Lambda(\Gamma)$.

Let us come back to the equality $\mathcal{L}_{\delta}(\varphi)=\mathrm{e}^{i \theta} \varphi$; since the modulus of $\varphi / h$ is constant on $\Lambda(\Gamma)$ one obtains by convexity $\varphi(a \cdot x) / h(a \cdot x)=\mathrm{e}^{i \theta} \varphi(x) / h(x)$ for any $a \in \Gamma_{j}^{*}$ and any $x \in \Lambda_{i}, i \neq j$. Fix $a \in \Gamma_{j}^{*}$; for any pair of points $x, x^{\prime}$ in $\Lambda(\Gamma)-\Lambda_{j}$, the sequences $a^{n} \cdot x$ and $a^{n} \cdot x^{\prime}$ converge to the attractive fixed point of $a$ and by continuity once again, we get

$$
\mathrm{e}^{i \theta} \frac{\varphi(x)}{h(x)}=\mathrm{e}^{i \theta} \frac{\varphi\left(x^{\prime}\right)}{h\left(x^{\prime}\right)} .
$$

The function $\varphi$ is thus proportional to $h$ and $\mathrm{e}^{i \theta}=1$; in other words, 1 is the unique eigenfunction of $\mathcal{L}_{\delta}$ with modulus 1 and the corresponding eigenspace is $\mathbb{C} \cdot h$.

When $L=2$, setting $\bar{\Lambda}_{i}=\Lambda(\gamma) \cap F_{i}$ for $i=1,2$, one gets $\mathcal{L}_{\delta}\left(h 1_{\bar{\Lambda}_{1}}\right)=h 1_{\bar{\Lambda}_{2}}$, $\mathcal{L}_{\delta}\left(h 1_{\bar{\Lambda}_{2}}\right)=h 1_{\bar{\Lambda}_{1}}$ and the operator $\mathcal{L}_{\delta}^{2}$ acts on each space $L_{\alpha}\left(\bar{\Lambda}_{1}\right)$ and $L_{\alpha}\left(\bar{\Lambda}_{2}\right)$. If $\varphi \in L_{\alpha}$ satisfies the equality $\mathcal{L}_{\delta} \varphi=\mathrm{e}^{i \theta} \varphi$, then $\mathcal{L}_{\delta}\left(\varphi 1_{\bar{\Lambda}_{j}}\right)=\mathrm{e}^{2 i \theta} \varphi 1_{\bar{\Lambda}_{j}}$ for $j=1,2$; the same argument than above leads to $\mathrm{e}^{2 i \theta}=1$ and $\varphi 1_{\bar{\Lambda}_{j}}=h 1_{\bar{\Lambda}_{j}}$. Consequently, either $\mathrm{e}^{i \theta}=1$ and $\varphi \in \mathbb{C} \cdot h$ or $\mathrm{e}^{i \theta}=-1$ and $\varphi \in \mathbb{C} \cdot h\left(1_{\bar{\Lambda}_{1}}-1_{\bar{\Lambda}_{2}}\right)$. This achieves the proof of Proposition III.4.

TOME $134-2006-\mathrm{N}^{\mathrm{O}} 1$ 
III-d. The Central Limit Theorem for the geodesic flow. - We will now use the preceding formalism to show a Central Limit Theorem for ( regular) additive functionals of the geodesic flow.

THEOREM III.5. - Let $X$ be a Hadamard manifold of pinched strictly negative curvature and $\Gamma=\Gamma_{1} * \cdots * \Gamma_{L}$ be a Schottky product of Kleinian groups acting on $X$ and satisfying the critical gap hypothesis. Let $M=X / \Gamma$ be the quotient manifold and $m$ the Patterson-Sullivan probability measure on $T^{1} M$. For any bounded and Hölder function $\Phi: T^{1} M \rightarrow \mathbb{R}$, the quantity

$$
\int_{T^{1} M} \frac{1}{t}\left(\int_{0}^{t}\left(\Phi\left(g_{s} v\right)-m(\Phi)\right) d s\right)^{2} m(\mathrm{~d} v)
$$

converges to a constant $\sigma_{\Phi}^{2}$. One has $\sigma_{\Phi}^{2}=0$ if and only if $\Phi$ is a coboundary, i.e. if $\Phi$ is the derivate in the direction of the flow of some Borel function defined on $T^{1} M$.

- When $\sigma_{\Phi}^{2} \neq 0$, the process $X_{t}(v)=\int_{0}^{t} \Phi\left(g_{s} v\right) d s$ satisfies the Central Limit Theorem: for any $a \in \mathbb{R}$

$$
m\left\{v: \frac{X_{t}(v)-t m(\Phi)}{\sigma_{\Phi} \sqrt{t}}>a\right\} \longrightarrow \pi([a,+\infty[)
$$

when $t \rightarrow+\infty$, where $\pi$ is a standard Gaussian law $\mathcal{N}(0,1)$ on $\mathbb{R}$.

- When $\sigma_{\Phi}^{2}=0$, the process $\left(\left(X_{t}-t m(\Phi)\right) / \sqrt{t}\right)_{t}$ tends to 0 in probability.

REMARK. - We cannot show that the CLT holds when $\Phi$ is only assumed to be in $L^{2}(m)$ (and Hölder continuous). In the course of the proof, we shall see that the boundedness condition can be relaxed up to a certain point. Analogous results in different set-ups have been obtained by various authors. The proof given here is simpler than Ratner's [32] original one, and is inspired by the works of Guivarc'h \& Hardy [17] and Guivarc'h \& Le Jan [18].

Proof. - For any vector $v$ in the subset $\Omega^{\prime}$ of full measure in the non-wandering set $\Omega$, there exist a unique lift $(y, x, r)$ of $v \in T^{1} M$ which belongs to the domain $D_{\ell}$ (see Section III-a); we will set $v=(y, x, r)$. The geodesic segment $\{(y, x, r-s) ; 0 \leq s \leq t\}$ will be cut into pieces, according to the values of the Birkhoff sums

$$
S_{n} \ell(x)=\ell(x)+\ell(T x)+\cdots+\ell\left(T^{n-1} x\right) .
$$

For a given $t$, we then define the process $N_{t}(v)$ by

$$
N_{t}(v)=\max \left\{n \geq 0 ; S_{n} \ell(x)<t+r\right\} .
$$

When the context is clear (i.e. when $t$ and $v$ are fixed) we shall often denote $S_{N_{t}(v)} \ell(x)$ simply by $S_{N}$. We shall decompose the proof into four steps. In the first one, we reduce the study of the process $X_{t}$ to the study of random Birkhoff sums over the dynamical system $(\Lambda, T, \nu)$. The second step gives the CLT for Birkhoff sums on this system. In the third one, we give a limit theorem for the 
process $N_{t}(v)$ and prove the above theorem in the non degenerated case. In the last step, we analyse the asymptotic variance $\sigma_{\Phi}^{2}$.

Without loss of generality, we will assume that $\Phi$ is $m$-centered, i.e.

$$
m(\Phi)=0 .
$$

Step 1. Reduction to the dynamical system $(\Lambda, T, \nu)$. — We first establish the following

Proposition III.6. - If $t>0$, let $N_{t}: \Lambda \rightarrow \mathbb{N}$ be the process defined for $v=(y, x, r) \in D_{\ell}$ by

$$
N_{t}(v)=\max \left\{n \geq 0 ; S_{n} \ell(x) \leq r+t\right\} .
$$

If the function $\Phi$ is Hölder continuous on $T^{1} M$ with exponent $\beta \leq 1$, there exists a Borel function $A: T^{1} M \rightarrow \mathbb{R}$ and a Hölder continuous function $\phi: \Lambda \rightarrow \mathbb{R}$ such that for any $v=(y, x, r) \in \Lambda$ one has, up to a uniformly bounded term:

$$
Y_{t}(v)=A\left(v_{t}\right)-A(v)+\sum_{k=0}^{N_{t}(v)-1} \phi\left(T^{k} x\right) .
$$

Moreover, there exists a constant $C>0$ such that

i) $|\phi(x)| \leq C \ell(x)+C$,

ii) $\left|\phi(\gamma \cdot x)-\phi\left(\gamma \cdot x^{\prime}\right)\right| \leq C\left|x-x^{\prime}\right|^{\frac{1}{2} \beta}$ for any $x, x^{\prime} \in \Lambda(\Gamma) \cap F_{i}$ and $\gamma \in \Gamma_{j}, j \neq i$.

Proof. - Let $\widetilde{\Phi}$ be the $\Gamma$-invariant lift of $\Phi$ to $T^{1} X$. For a vector $v=(y, x, r)$ in $D_{\ell}$, one has

$$
\begin{aligned}
X_{t}(v) & =\int_{0}^{t} \widetilde{\Phi}(y, x, r-s) \mathrm{d} s \\
& =-\int_{0}^{r} \widetilde{\Phi}(y, x,-s) \mathrm{d} s+\int_{0}^{S_{N}} \widetilde{\Phi}(y, x,-s) \mathrm{d} s+\int_{S_{N}}^{t+r} \widetilde{\Phi}(y, x,-s) \mathrm{d} s .
\end{aligned}
$$

Let $A(v)$ be the function defined by $A(v)=\int_{0}^{r} \widetilde{\Phi}(y, x,-s) \mathrm{d} s$. Since $\widetilde{\Phi}$ is $T_{\ell^{-}}$ invariant,

$$
\int_{S_{N}}^{t+r} \widetilde{\Phi}(y, x,-s) \mathrm{d} s=A\left(g_{t} v\right)
$$

On the other hand, we have

$$
\begin{aligned}
\int_{0}^{S_{N}} \widetilde{\Phi}(y, x,-s) \mathrm{d} s & =\sum_{k=0}^{N_{t}(v)-1} \int_{0}^{\ell\left(T^{k} x\right)} \widetilde{\Phi}\left(y, x,-s-S_{k} \ell(x)\right) \mathrm{d} s \\
& =\sum_{k=0}^{N_{t}(v)-1} \int_{0}^{\ell\left(T^{k} x\right)} \widetilde{\Phi}\left(\bar{T}^{\ell}(y, x),-s\right) \mathrm{d} s=\sum_{k=0}^{N_{t}(v)-1} \bar{\phi}\left(\bar{T}^{\ell}(y, x)\right)
\end{aligned}
$$

TOME $134-2006-\mathrm{N}^{\mathrm{O}} 1$ 
where we have set $\bar{\phi}(y, x)=\int_{0}^{\ell(x)} \widetilde{\Phi}(y, x, s) \mathrm{d} s$. The function $\bar{\phi}$ is Borel regular and satisfies the inequality $|\bar{\phi}(y, x)| \leq\|\Phi\|_{\infty} \ell(x)$ by the boundedness of $\widetilde{\Phi}$; it is thus integrable with respect to $\bar{\nu}$ on $\bar{\Lambda}$. Moreover, since the Patterson-Sullivan measure $m$ on $T^{1} M$ identifies with the measure $\bar{\nu} \otimes d s$ on the domain $\bar{D}_{\ell}$, we have

$$
\int_{\bar{\Lambda}} \bar{\phi}(y, x) \mathrm{d} \bar{\nu}(y, x)=\int_{\bar{\Lambda}_{\ell}} \widetilde{\Phi}(y, x, s) \mathrm{d} m(y, x, s)=0 .
$$

One achieves the proof of Proposition III.6 using the following lemma which brings the study to the factor system $(\Lambda, T, \nu)$ and relies on standard arguments (see [5] and also [2, lemme 4.3]) for a precise statement developed in a very close set-up):

Lemma III.7. - Assume that $\Phi$ is Hölder continuous on $T^{1} M$ with exponent $\beta$. There exist Borel functions $\phi: \Lambda \rightarrow \mathbb{C}$ and $\psi: \bar{\Lambda} \rightarrow \mathbb{C}$ such that for any $(y, x) \in \bar{\Lambda}$,

$$
\bar{\phi}(y, x)=\phi(x)+\psi(y, x)-\psi \circ \bar{T}(y, x) .
$$

Moreover, there exists a constant $C>0$ such that

i) $|\phi(x)| \leq C \ell(x)+C$;

ii) $\left|\phi(\gamma \cdot x)-\phi\left(\gamma \cdot x^{\prime}\right)\right| \leq C\left|x-x^{\prime}\right|^{\frac{1}{2} \beta}$ for any $x, x^{\prime} \in \bar{\Lambda}(\Gamma) \cap F_{i}$ and $\gamma \in \Gamma_{j}, j \neq i$;

iii) the function $\psi$ is bounded.

One can finally decompose the process $X_{t}(v)$ as

$$
X_{t}(v)=\sum_{k=1}^{N_{t}(v)} \phi\left(T^{k} x\right)+R_{t}(v)
$$

where $\phi$ satisfies the conclusions of the above lemma and $\int_{\Lambda} \phi \mathrm{d} \nu=0$, and the remainder term $R_{t}(v)$ is of the form $A\left(g_{t} v\right)-A(v)+\psi(y, x)-\psi\left(\bar{T}^{N}(y, x)\right)$ with $N=N_{t}(v)$. Since $A$ is integrable with respect to $m$ and $\psi$ bounded, $\left(R_{t} / \sqrt{t}\right)_{t}$ converges almost surely to 0 , so that it now suffices to study the behavior in distribution of the random sum $\sum_{k=0}^{N_{t}(v)-1} \phi\left(T^{k} x\right)$.

Step 2. The Central Limit Theorem for Birkhoff sums on $(\Lambda, T, \nu)$. - We prove the following statement:

Proposition III.8. - Let $\mathbf{f}$ be a Borel function from $\Lambda(\Gamma)$ to $\mathbb{C}$ such that

i) $|\mathbf{f}(x)| \leq C \ell(x)+C$ for all $x \in \Lambda$;

ii) $|\mathbf{f}(\gamma \cdot x)-\mathbf{f}(\gamma \cdot y)| \leq C|x-y|^{\alpha}$ for any $x, y \in \Lambda_{\Gamma} \cap F_{i}$ and $\gamma \in \Gamma_{j}, j \neq i$.

Then, one has $\nu\left(\mathbf{f}^{2}\right)<+\infty$ and the sequence $\left(\nu\left(\left(S_{n} \mathbf{f}-n \nu(\mathbf{f})\right)^{2}\right) / n\right)_{n}$ converges to a constant $\sigma_{\mathbf{f}}^{2}$; furthermore, $\sigma_{\mathbf{f}}^{2}=0$ if and only if $\mathbf{f}$ is a T-coboundary in $L_{\alpha}$, i.e. if there exists a function $f \in L_{\alpha}$ such that $\mathbf{f}=\nu(\mathbf{f})+f \circ T-f$.

BULletin DE LA SOCiÉtÉ MATHÉmATiQUe DE FRANCE 
- When $\sigma_{\mathbf{f}}^{2}>0$ one has

$$
\frac{1}{\sigma_{\mathbf{f}} \sqrt{n}}\left(S_{n} \mathbf{f}(x)-n \nu(\mathbf{f})\right) \stackrel{\mathcal{L}}{\longrightarrow} \mathcal{N}(0,1),
$$

the point $x$ being distributed among the probability measure $\nu$.

- Otherwise, $a_{n} S_{n} \mathbf{f} \stackrel{\mathcal{L}}{\rightarrow} 0$ for any sequence $\left(a_{n}\right)_{n}$ which tends to 0 .

Sketch of the proof. - This is a well-known result (see [17], [21]); we just recall here the idea of the proof with some complements which will be of interest in the Step 4. Under hypothesis i), one has $\nu\left(|\mathbf{f}|^{k}\right)<+\infty$ for any $k \geq 0$; without loss of generality, we will assume $\nu(\mathbf{f})=0$.

Using the duality between $\mathcal{L}_{\delta}$ and the transformation $T$, we have for all $u \in \mathbb{R}$

$$
\int_{\Lambda} \exp \left(i u \frac{S_{n} \mathbf{f}(x)}{\sqrt{n}}\right) \mathrm{d} \nu(x)=\int_{\Lambda} \mathcal{L}_{\delta}\left[\mathrm{e}^{i u \mathbf{f}} \mathcal{L}_{\delta}\left[\mathrm{e}^{i u \mathbf{f}}\left[\cdots \mathcal{L}_{\delta} \mathrm{e}^{i u \mathbf{f}} h\right] \cdots\right]\right](x) \sigma(\mathrm{d} x) .
$$

This leads us to introduce the Fourier operator $\mathcal{L}_{\delta, u}$ acting on $L_{\alpha}$ by

$$
\mathcal{L}_{\delta, u} \psi=\mathcal{L}_{\delta}\left(\mathrm{e}^{i u \mathbf{f}} \psi\right)
$$

so that we get

$$
\int_{\Lambda} \exp \left(i u \frac{S_{n} \mathbf{f}(x)}{\sqrt{n}}\right) \nu(\mathrm{d} x)=\int_{\Lambda} \mathcal{L}_{\delta, u / \sqrt{n}}^{n} h(x) \sigma(\mathrm{d} x) .
$$

Note that $\mathcal{L}_{\delta, 0}$ coincides with $\mathcal{L}_{\delta}$ and that, by hypothesis ii), the operator $\mathcal{L}_{\delta, u}$ acts on $L_{\alpha}$ for any $u \in \mathbb{R}$; furthermore the function $u \mapsto \mathcal{L}_{\delta, u}$ is $C^{\infty}$ regular from $\mathbb{R}$ into the space $\mathcal{L}\left(L_{\alpha}\right)$ of linear operators on $L_{\alpha}$.

Assume now $L>2$, i.e. $\Gamma$ contains at least three Schottky factors; the case $L=2$ is a little more delicate since $\mathcal{L}_{\delta, u}$ has two dominant eigenvalues whose regularity in $u$ has to be controlled, we refer to [21] for a complete proof. By Proposition III.4, there exists a linear operator $R$ on $L_{\alpha}$ with spectral radius $<1$ such that

$$
\mathcal{L}_{\delta}(.)=\sigma(.) h+R(.) .
$$

By perturbation theory, there exists an open neigbourhood $U$ of 0 and $C^{2}$ functions $u \mapsto \lambda_{u}$ from $U$ to $\mathbb{C}, u \mapsto h_{u}$ from $U$ to $L_{\alpha}, u \mapsto \sigma_{u}($.$) from U$ to the dual space $L_{\alpha}^{\prime}$ of $L_{\alpha}$ and $u \mapsto R_{u}$ from $U$ to $\mathcal{L}\left(L_{\alpha}\right)$ such that

$$
\mathcal{L}_{\delta, u}(.)=\lambda_{u} \sigma_{u}(.) h_{u}+R_{u}(.)
$$

with $\lambda_{0}=1, h_{0}=h$ and $R_{0}=R$. The functions $u \mapsto h_{u}$ and $u \mapsto \sigma_{u}($.$) are$ determined up to a multiplicative constant, one may thus normalize $h_{u}$ and $\sigma_{u}$ in such a way that $\sigma\left(h_{u}\right)=1$ and $\sigma_{u}\left(h_{u}\right)=1$. One has

$$
\mathcal{L}_{\delta, u}^{n} h(x)=\lambda_{u}^{n} \sigma_{u}(h) h_{u}(x)+R_{u}^{n} h(x) .
$$

TOME $134-2006-\mathrm{N}^{\mathrm{O}} 1$ 
The function $u \mapsto \sigma\left(R_{u}^{n} h\right)$ is $C^{2}$ and vanishes at 0 ; furthermore, $\sigma_{u}(h)=1+o(u)$ and there exists $\eta>0$ and $C>0$ such that $\left\|R_{u}^{n}\right\|_{\alpha} \leq C(1-\eta)^{n}$ for any $u$ in $U$; so

$$
\sigma\left(\mathcal{L}_{\delta, u}^{n} h\right)=\lambda_{u}^{n}+u \epsilon_{n}(u) \quad \text { with } \quad\left|\epsilon_{n}(u)\right| \leq C\left(\left|\lambda_{u}\right|^{n}+(1-\eta)^{n}\right) .
$$

The control of the dominant term $\lambda_{u}^{n}$ relies on the following

LEMmA III.9. - One has $\lambda_{0}^{\prime}=0$ and $\lambda_{0}^{\prime \prime}=-\nu\left(\mathbf{f}^{2}\right)+2 i \nu\left(\mathbf{f} h_{0}^{\prime}\right)$. Furthermore $\lambda_{0}^{\prime \prime} \in \mathbb{R}^{-}$and the equality $\lambda_{0}^{\prime \prime}=0$ holds if and only if $\mathbf{f}$ is a coboundary in $L_{\alpha}$. At last, the sequence $\left(n \lambda_{0}^{\prime \prime}+\nu\left(\left(S_{n} \mathbf{f}\right)^{2}\right)\right)_{n \geq 1}$ is bounded.

Proof. - Differentiating the two sides of the equality $\lambda_{u}=\sigma\left(\mathrm{e}^{i u \mathbf{f}} h_{u}\right)$ yields to

$$
\lambda_{u}^{\prime}=i \sigma\left(\mathrm{e}^{i u \mathbf{f}} \mathbf{f} h_{u}\right)+\sigma\left(\mathrm{e}^{i u \mathbf{f}} h_{u}^{\prime}\right) .
$$

Letting $u=0$ one gets $\lambda_{0}^{\prime}=i \sigma(\mathbf{f} h)+\sigma\left(h_{0}^{\prime}\right)$; the normalisation condition $\sigma\left(h_{u}\right)=1$ implies $\sigma\left(h_{0}^{\prime}\right)=0$ and so $\lambda_{0}^{\prime}=i \nu(\mathbf{f})=0$. Differentiating again the two sides of the equality (6) and letting $u=0$ we get

$$
\lambda_{0}^{\prime \prime}=-\nu\left(\mathbf{f}^{2}\right)+2 i \sigma\left(\mathbf{f} h_{0}^{\prime}\right) .
$$

Conjugating the two sides in the equality $\mathcal{L}_{\delta, u} h_{u}=\lambda_{u} h_{u}$, one gets $\mathcal{L}_{\delta,-u} \bar{h}_{u}=$ $\bar{\lambda}_{u} \bar{h}_{u}$ and so $\lambda_{-u}=\bar{\lambda}_{u}$ and $h_{-u}=\bar{h}_{u}$; the derivate at 0 of the function $u \mapsto h_{u}$ is thus purely imaginary valued, which implies that $h_{0}^{\prime}=i h f$ for some real valued function $f$ in $L_{\alpha}$. Differentiating the equality $\mathcal{L}_{\delta, u} h_{u}=\lambda_{u} h_{u}$ and letting $u=0$, one gets $\mathcal{L}_{\delta}((\mathbf{f}-f) h)=h f$ and so $\nu(\mathbf{f} f \circ T)=\nu\left(f^{2}\right)-\nu(f \cdot f \circ T)$; this readily implies

$$
\lambda_{0}^{\prime \prime}=-\left(\nu\left(\mathbf{f}^{2}\right)+2 \nu(\mathbf{f} f)\right)=-\nu\left((\mathbf{f}+f-f \circ T)^{2}\right) .
$$

Set $\sigma_{\mathbf{f}}=\sqrt{\nu\left((\mathbf{f}+f-f \circ T)^{2}\right)}$ and remark that $\sigma_{\mathbf{f}}=0$ if and only if $\mathbf{f}$ is a coboundary in $L_{\alpha}$. To prove the last assertion of Lemma III.9, let us remark that $\sigma\left(\mathcal{L}_{\delta, u}^{n} h\right)=\nu\left(\mathrm{e}^{i u S_{n} \mathbf{f}}\right)$ and so $\sigma\left(\left(\mathcal{L}_{\delta, u}^{n} h\right)_{\mid u=0}^{\prime \prime}\right)=-\nu\left(\left(S_{n} \mathbf{f}\right)^{2}\right)$. On the other hand, $\mathcal{L}_{\delta, u}^{n} h=\lambda_{u}^{n} \sigma_{u}(h) h_{u}+R_{u}^{n} h$; an elementary calculus leads to

$$
\sigma\left(\left(\mathcal{L}_{\delta, u}^{n} h\right)_{\mid u=0}^{\prime \prime}\right)=n \lambda_{0}^{\prime \prime}+\sigma\left(\frac{\mathrm{d}^{2}}{\mathrm{~d} u^{2}}\left(\sigma_{u}(h) h_{u}+R_{u}^{n} h\right)\right)_{u=0}
$$

the last term being bounded in $n$. This achieves the proof of Lemma III.9.

Let us now achieve the proof of Proposition III.8. If $\mathbf{f}$ is not a coboundary, one gets

$$
\sigma\left(\mathcal{L}_{\delta, u}^{n} h\right)=\left(1-\frac{1}{2} \sigma_{\mathbf{f}}^{2} u^{2}+u^{2} o(u)\right)^{n}+u \epsilon_{n}(u)
$$

it follows immediately

$$
\lim _{n \rightarrow+\infty} \sigma\left(\mathcal{L}_{\delta, u / \sqrt{n}}^{n} h\right)=\exp \left(-\frac{1}{2} \sigma_{\mathbf{f}}^{2} u^{2}\right) .
$$

This is the expected result when $\mathbf{f}$ is not a coboundary. The second assertion of the proposition is obvious.

BULLETIN DE LA SOCIÉtÉ MATHÉMATIQUE DE FRANCE 
REMARK. - Conditions i) and ii) of Proposition III.8 may be obviously relaxed. In fact we only need that the function $u \mapsto \mathcal{L}_{\delta, u}$ is $C^{2}$-regular. It seems to be not possible (neither natural) to express this condition directly in term of the function f. In Subsection IV-d, we will apply Proposition III.8 to another class of functions $\mathbf{f}$ than the one considered here.

In the sequel, we will apply Proposition III.8 to the function $\phi$ associated with $\Phi$ (see Lemma III.7); the fact that the sequence $\left(n \lambda_{0}^{\prime \prime}+\nu\left(\left(S_{n} \phi\right)^{2}\right)\right)_{n \geq 1}$ is bounded will be important to express the variance $\sigma_{\phi}^{2}$ in terms of the function $\Phi$. In fact we will need more than this estimation; we have the

LEMma III.10. - Assume that hypotheses of Proposition III.8 hold and that $\nu(\mathbf{f})=0$. Then, for any $k \geq 1$ there exists a constant $C_{k}>0$, such that

$$
\forall n \geq 1, \quad \nu\left(\left(S_{n} \mathbf{f}\right)^{2 k}\right) \leq C_{k} n^{k} .
$$

Proof. - This lemma is close to Lemma 3.2 in [32]; the proof proposed here is new and quite simpler. For any $n, k \geq 1$, one has

$$
\left|\sigma\left(\left(\mathcal{L}_{\delta, u}^{n} h\right)_{\mid u=0}^{(2 k)}\right)\right|=\nu\left(\left(S_{n} \mathbf{f}\right)^{2 k}\right) .
$$

Since $\mathcal{L}_{\delta, u}^{n} h=\lambda_{u}^{n} \sigma_{u}(h) h_{u}+R_{u}^{n} h$ and the spectral radius of $R_{u}$ is $<1$ for $u$ small enough, it suffices to prove that the sequence $\left|\left(\lambda_{u}^{n}\right)^{(2 k)}\right|_{u=0} \mid / n^{k}$ is bounded in $n$. Recall that $\lambda_{u}$ admits the local expansion $\lambda_{u}=a_{0}+a_{1} u+a_{2} u^{2}+\cdots$ with $a_{0}=1$ and $a_{1}=0$; in particular, the term $\left.\left(\lambda_{u}^{n}\right)^{(2 k)}\right|_{u=0}$, which is also the $2 k$-th coefficient of the local expansion of $\lambda_{u}^{n}$, is equal to

$$
a_{n}^{(2 k)}=\sum_{\substack{\ell_{1}, \ldots \ell_{n} \geq 0 \\ \sum_{i} \ell_{i}=2 k}} \frac{(2 k) !}{\ell_{1} ! \ldots \ell_{n} !} a_{\ell_{1}} \cdots a_{\ell_{n}} .
$$

Note that none of the $\ell_{i}$ is equal to 1 in the previous sum since $a_{1}=0$; the term $\left|a_{n}^{(2 k)}\right|$ is thus less than $(2 k) ! A_{k}^{2 k} \sharp I_{n, k}$ with $A_{k}=\sup _{0 \leq i \leq 2 k}\left|a_{i}\right|$ and

$$
I_{n, k}=\left\{\left(\ell_{1}, \ldots, \ell_{n}\right) ; 0 \leq \ell_{i} \leq 2 k, \sum_{i} \ell_{i}=2 k, \ell_{i} \neq 1\right\} .
$$

A simple inductive argument show that there exists a universal constant $C>0$ such that $\sharp I_{n, k} \leq C n^{k}$ for any $n, k \geq 1$. This achieves the proof of the lemma.

Step 3. Proof of the Central Limit Theorem for the process $\left(X_{t}\right)$. - - One may apply Proposition III.8 to the function $\ell$ itself. Note that $\sigma_{\ell}^{2}>0$; otherwise $\ell-\nu(\ell)$ should be a coboundary in $L_{\alpha}$ and so $\ell$ should be bounded. Contradiction.

Using thus classical techniques coming from the probability theory, one obtains the following

TOME $134-2006-\mathrm{N}^{\mathrm{O}} 1$ 
Corollary III.11. - One has $\lim _{t \rightarrow+\infty} N_{t}(v)=+\infty$ for $m$-almost all $v$ and the process

$$
\left(\sqrt{t}\left(\frac{N_{t}(.)}{t}-\frac{1}{\nu(\ell)}\right)\right)_{t>0}
$$

converges in law to a Gaussian law $\mathcal{N}\left(0, \sigma_{\ell}^{2} / \nu(\ell)^{3}\right)$.

Proof. - To deduce this corollary from Proposition III.8, fix $a \in \mathbb{R}$ and set $a_{t}=[a \sqrt{t}+t / \nu(\ell)]$; one has

$$
\begin{aligned}
m\left\{v ; \sqrt{t}\left(N_{t} / t-1 / \nu(\ell)\right) \leq a\right\} & =m\left\{v=(y, x, r) ; S_{a_{t}}>t+r\right\} \\
& =\left\{v ; \frac{S_{a_{t}}-a_{t} \nu(\ell)}{\sigma_{\ell} \sqrt{a_{t}}}>\frac{t+r-a_{t} \nu(\ell)}{\sigma_{\ell} \sqrt{a_{t}}}\right\} .
\end{aligned}
$$

Since $\left(t+r-a_{t} \nu(\ell)\right) / \sigma_{\ell} \sqrt{a_{t}}=-a \nu(\ell)^{3} / \sigma_{\ell}(1+O(1 / \sqrt{t}))$ and $r / \sqrt{a_{t}} \rightarrow 0$ in probability, one has finally

$$
\lim _{t \rightarrow+\infty} m\left\{v ; \sqrt{t}\left(N_{t} / t-1 / \nu(\ell)\right) \leq a\right\}=\frac{1}{\sqrt{2 \pi}} \int_{-\infty}^{a \nu(\ell)^{3} / \sigma_{\ell}} \mathrm{e}^{-\frac{1}{2} x^{2}} \mathrm{~d} x
$$

which is the expected convergence.

We now prove Theorem III.5. For any $v=(y, x, r) \in D_{\ell}$, one has $X_{t}(v)=$ $\sum_{k=0}^{N_{t}(v)-1} \phi\left(T^{k} x\right)+R_{t}(v)$, the function $\phi$ satisfying the hypotheses of Proposition III.8. Set $\sigma_{\Phi}^{2}=\sigma_{\phi}^{2} / \nu(\ell)$ where $\sigma_{\phi}^{2}=\lim _{n \rightarrow+\infty} \nu\left(\left(S_{n} \phi-n \nu(\phi)\right)^{2}\right) / n$ is the variance associated with $\phi$.

Assume first $\sigma_{\phi}^{2} \neq 0$. The term $(1 / \sqrt{t}) R_{t}(v)$ is not relevant since it tends to 0 in probability. To control $(1 / \sqrt{t}) S_{N_{t}(v)} \phi(x)$ we write $N_{t}(v)=n_{t}+\left(N_{t}(v)-n_{t}\right)$ with $n_{t}=[t / \nu(\ell)]$; by Proposition III.8, the sequence $\left((1 / \sqrt{t}) S_{n_{t}} \phi\right)_{t}$ converges in distribution to a Gaussian law $\mathcal{N}\left(\nu(\phi), \sigma_{\phi}^{2}\right)$. On the other hand, for any non negative constants $K$ and $\epsilon$, one may decompose the set $\left\{v \in D_{\ell}\right.$; $\left.\left|S_{N_{t}(v)} \phi(x)-S_{n_{t}} \phi(x)\right| \geq \epsilon \sqrt{t}\right\}$ into $A_{t}+B_{t}$ where

$$
\begin{aligned}
& A_{t}=\left\{v \in D_{\ell} ;\left|S_{N_{t}(v)} \phi(x)-S_{n_{t}} \phi(x)\right| \geq \epsilon \sqrt{t},\left|N_{t}(v)-n_{t}\right| \geq K \sqrt{t}\right\}, \\
& B_{t}=\left\{v \in D_{\ell} ;\left|S_{N_{t}(v)} \phi(x)-S_{n_{t}} \phi(x)\right| \geq \epsilon \sqrt{t},\left|N_{t}(v)-n_{t}\right|<K \sqrt{t}\right\} .
\end{aligned}
$$

One has obviously $A_{t} \subset\left\{v \in D_{\ell} ;\left|N_{t}(v)-n_{t}\right| \geq K \sqrt{t}\right\}$; the tightness of the process $\left(\frac{N_{t}-n_{t}}{\sqrt{t}}\right)_{t}$ (which is a direct consequence of corollary III.11) implies $\lim \sup _{K \rightarrow+\infty} \lim \sup _{t \rightarrow+\infty} m\left(A_{t}\right)=0$. On the other hand

$$
\begin{aligned}
B_{t} & =\left\{v \in D_{\ell} ;\left|S_{N_{t}(v)} \phi(x)-S_{n_{t}} \phi(x)\right| \geq \epsilon \sqrt{t},\left|N_{t}(v)-n_{t}\right|<K \sqrt{t}\right\} \\
& \subset\left\{v \in D_{\ell} ; 1 / \sqrt{t} \sup _{0 \leq k \leq K \sqrt{t}}\left|S_{k} \phi(x)\right| \geq \epsilon\right\} .
\end{aligned}
$$


To conclude one uses the following lemma which extends a previous result due to Y. Guivar'ch and Y. Le Jan [19]:

Lemma III.12. - For any Borel function $\phi: \Lambda \rightarrow \mathbb{C}$, set

$$
M_{n} \phi=\sup _{0 \leq k \leq n}\left|S_{k} \phi\right| .
$$

Assume that $\phi$ satisfies the hypotheses of Proposition III.8 and $\nu(\phi)=0$. Then

i) the sequence $\left(n^{-1} M_{n} \phi\right)_{n}$ converges to 0 in probability.

ii) for any $s>0$, one has

$$
\nu\left(\left(\frac{1}{n} \sup _{0 \leq k \leq n}\left|S_{k} \phi\right|\right)^{s}\right) \leq 1+2 \nu\left(\left|\frac{S_{n} \phi}{n}\right|^{s}\right)<+\infty .
$$

Proof. - It relies on some techniques developed in probability theory. Remark that the operator $Q: \varphi \mapsto h^{-1} \mathcal{L}_{\delta}(h \varphi)$ is a Markov operator. We may thus introduce a Markov chain $\left(X_{n}\right)_{n \geq 0}$ on $\Lambda$ with transition operator $Q$ and we denote by $\left(\Lambda^{\otimes \mathbb{N}}, \mathcal{B}(\Lambda)^{\otimes \mathbb{N}},\left(\mathbb{P}_{x}\right)_{x \in \Lambda}\right)$ the canonical probability space associated with $\left(X_{n}\right)_{n \geq 0}$; in particular, for any probability measure $m$ on $\Lambda$ one denotes by $\mathbb{P}_{m}$ the probability measure on $\Lambda^{\otimes \mathbb{N}}$ so that the law of $X_{0}$ equals $m$, and by $\mathbb{E}_{m}$ the associated expectation. In this context, the duality between $\mathcal{L}_{\delta}$ and $T$ readily implies that for any bounded Borel function $\Psi$ on $\Lambda^{n}$, one has

$$
\int_{\Lambda} \Psi\left(x, T x, \ldots, T^{n-1} x\right) \nu(\mathrm{d} x)=\mathbb{E}_{\nu}\left[\Psi\left(X_{n-1}, \ldots, X_{0}\right)\right] .
$$

In particular, for any $n \geq 1$, the functions $S_{n} \phi$ and $n^{-1} \sup _{0<k<n}\left|S_{k} \phi\right|$ can be considered as random variables on the probability space $(\bar{\Lambda}, \overline{\mathcal{B}}(\Lambda), \nu)$ whose law is respectively the same as the one of the random variables $S_{n}=$ $\phi\left(X_{0}\right)+\cdots+\phi\left(X_{n-1}\right)$ and $M_{n}=\sup _{0 \leq k \leq n}\left|S_{k}\right|$ defined on $\left(\Lambda^{\otimes \mathbb{N}}, \mathcal{B}(\Lambda)^{\otimes \mathbb{N}}, \mathbb{P}_{\nu}\right)$. Recall that $\mathcal{L}_{\delta}$ acts on $L_{\alpha}$, that it is quasi-compact on this space with 1 as a simple and isolated dominant eigenvalue when $L>2$ (when $L=2, \mathcal{L}_{\delta}^{2}$ acts on $L_{\alpha}\left(\bar{\Lambda}_{1}\right)$ and on $L_{\alpha}\left(\bar{\Lambda}_{2}\right)$, and the same conclusion holds on each of these two spaces). By the argument developed in the proof of Proposition III.8, one may proves that, for any $t \in \mathbb{R}$, the sequence $\left.\left(\mathcal{L}_{\delta, u / \sqrt{n}}^{n} h\right)(x)\right)$ converges, uniformly in $x$ in $\Lambda$, either to $\exp \left(-\frac{1}{2} \sigma_{\phi} t^{2}\right)$ with $\sigma_{\phi}^{2}>0$ when $\phi$ is not a coboundary, or to 0 otherwise. By a standard argument in probability theory, that proves that the sequence of random variables $\left(S_{n} / \sqrt{n}\right)_{n}$ converges in law according to the probability $\mathbb{P}_{x}$, uniformly in $x \in \Lambda$, which implies in particular that $\lim _{n \rightarrow+\infty} \sup _{x \in \Lambda} \mathbb{P}_{x}\left[\left|S_{n}\right| \geq n^{\eta} \epsilon\right]=0$ for any $\eta>\frac{1}{2}$ and $\epsilon>0$.

To prove the lemma, we will check that for such $\eta \in] \frac{1}{2}, 1[$ and any $c>0$, there exists $n_{1} \geq 1$ depending only on $\eta$ such that for any $n \geq n_{1}$ and $x \in \Lambda$ one has

$$
\mathbb{P}_{x}\left[\left|S_{n}\right| \geq n c-n^{\eta}\right] \geq \frac{1}{2} \mathbb{P}_{x}\left[M_{n} \geq n c\right] .
$$

TOME $134-2006-\mathrm{N}^{\mathrm{O}} 1$ 
The first assertion of Lemma III.12 is a direct consequence of this inequality. The second one follows from the fact that for $n^{1-\eta} \geq 2$ one has

$$
\begin{aligned}
\mathbb{E}_{x}\left[\left(\frac{M_{n}}{n}\right)^{s}\right] & \leq 1+\sum_{k \geq 1} \mathbb{P}_{x}\left[\left(\frac{M_{n}}{n}\right)^{s} \geq k\right] \\
& \leq 1+2 \sum_{k \geq 1} \mathbb{P}_{x}\left[\left(2 \frac{\left|S_{n}\right|}{n}\right)^{s} \geq k\right] \leq 1+2 \mathbb{E}_{x}\left[\left(2 \frac{\left|S_{n}\right|}{n}\right)^{s}\right] .
\end{aligned}
$$

the last term being finite by Lemma III.10.

To prove inequality (7), we fix $a, b \in \mathbb{R}^{*}$ and, for any $0 \leq k \leq n-1$ we consider the event $A_{k}=\left[\left|S_{0}\right|<a+b, \ldots,\left|S_{k-1}\right|<a+b,\left|S_{k}\right| \geq \bar{a}+b\right]$; these sets are pairwise disjoint, and, setting $S_{k}^{n}=\phi\left(X_{k}\right)+\cdots+\phi\left(X_{n-1}\right)$ one may write

$$
\begin{aligned}
\mathbb{P}_{x}\left[\left|S_{n}\right| \geq a\right] \geq \sum_{k=0}^{n-1} \mathbb{P}_{x}\left[A_{k} \cap\left[\left|S_{k}^{n}\right| \leq b\right]\right] & \geq \sum_{k=0}^{n-1} \int_{A_{k}} \mathbb{P}_{X_{k}(\omega)}\left[\left|S_{k}^{n}\right| \leq b\right] \mathbb{P}_{x}(\mathrm{~d} \omega) \\
& \geq \sum_{k=0}^{n-1} \mathbb{P}_{x}\left(A_{k}\right) \inf _{y \in \Lambda} \mathbb{P}_{y}\left[\left|S_{n-k}\right| \leq b\right]
\end{aligned}
$$

We will set $a=n c-n^{\eta}$ and $b=n^{\eta}$. Since $\mathbb{P}_{x}\left[\left|S_{n}\right| \leq n^{\eta}\right] \rightarrow 1$ uniformly in $x \in \Lambda$, there exists $n_{0}$ such that for $n-k \geq n_{0}$,

$$
\inf _{y \in \Lambda} \mathbb{P}_{y}\left[\left|S_{n-k}\right| \leq n^{\eta}\right] \geq \inf _{y \in \Lambda} \mathbb{P}_{y}\left[\left|S_{n-k}\right| \leq(n-k)^{\eta}\right] \geq \frac{1}{2} .
$$

On the other hand, for $n-k<n_{0}$ one has

$$
\mathbb{P}_{y}\left[\left|S_{n-k}\right| \leq n^{\eta}\right] \geq \mathbb{P}_{y}\left[M_{n_{0}} \leq n^{\eta}\right] \longrightarrow 1
$$

uniformly in $y \in \Lambda$ as $n \rightarrow+\infty$. There thus exists $n_{1}$ such that

$$
\mathbb{P}_{y}\left[\left|S_{n-k}\right| \leq n^{\eta}\right] \geq \frac{1}{2}
$$

for any $y \in \Lambda, n \geq n_{1}$ and $1 \leq k \leq n$. This gives the expected result since $\left[M_{n} \geq n c\right]$ is the disjoint union of the $A_{k}, 0 \leq k \leq n-1$.

Step 4. Analysis of the variance. - We simplify and extend here M. Ratner's arguments [32]. Let $\Phi: T^{1} M \rightarrow \mathbb{C}$ a bounded and Hölder continuous function such that $m(\Phi)=0$. We denote by $\bar{\phi}$ (resp. $\phi$ ) the associated Borel function defined on $\bar{\Lambda}$ (resp. on $\Lambda$ ); recall that $\bar{\nu}(\bar{\phi})=\nu(\phi)=0$. We have $\sigma_{\phi}^{2}=0$ if and only if $\phi$ is a coboundary in $L_{\alpha}$ : there exists $\varphi \in L_{\alpha}$ such that $\phi=\varphi-\varphi \circ T$. By Lemma III.6, this occurs if and only if $\bar{\phi}=F-F \circ \bar{T}$ for some Borel bounded function $F: \bar{\Lambda} \rightarrow \mathbb{C}$. If such a function $F$ exists then $\Phi$ is a coboundary for the geodesic flow on $T^{1} M$ : setting $\Phi^{*}(y, x, t)=-F(y, x)+\int_{0}^{t} \Phi(y, x, s) \mathrm{d} s$ (with the usual identification $(y, x, \ell(x)) \sim(\bar{T}(y, x), 0))$ one has, for any $r>0$ :

$$
\Phi_{r}=\Phi^{*} \circ g_{r}-\Phi^{*} \quad \text { where } \Phi_{r}(y, x, t)=\int_{0}^{r} \Phi(y, x, t+s) \mathrm{d} s
$$

BULLETIN DE LA SOCiÉTÉ MATHÉMATIQUe DE FRANCE 
(one easily checks that $\Phi^{*}(y, x, \ell(x))=\Phi^{*}(\bar{T}(y, x), 0)$ a.s. and that the above coboundary equation holds). Conversely, if $\Phi$ is a coboundary for the geodesic flow on $T^{1} M$ there exists a Borel function $\Phi^{*}: T^{1} M \rightarrow \mathbb{C}$ such that $\Phi_{r}=\Phi^{*} \circ g_{r}-\Phi^{*} m$-a.s. for any $r>0$, and the continuity of $\Phi$ implies $\partial \Phi^{*} / \partial t=\Phi m$-a.s. Setting $F(y, x)=-\Phi^{*}(y, x, 0)$ one thus gets $\bar{\phi}=F-F \circ \bar{T}$.

Let us now establish the fact that

$$
\int_{T^{1} M} \frac{X_{t}^{2}(v)}{t} m(\mathrm{~d} v) \longrightarrow \sigma_{\phi}^{2} / \nu(\ell)=\sigma_{\Phi}^{2}
$$

as $t \rightarrow+\infty$. Since the process $\left(X_{t}\right)_{t}$ satisfies the Central Limit Theorem with variance $\sigma_{\phi}^{2}$, it suffices in fact to establish the equi-integrability of the family $\left(X_{t}^{2} / t\right)_{t>0}$, i.e. that, for any $C>0$, the integral $\int_{\left[X_{t}^{2} \geq t C\right]} t^{-1} X_{t}^{2}(v) m(\mathrm{~d} v)$ tends to 0 as $C \rightarrow+\infty$, uniformly in $t>0$. Since

$$
\int_{\left[X_{t}^{2} \geq t C\right]} \frac{X_{t}^{2}(v)}{t} m(\mathrm{~d} v) \leq \frac{1}{C} \int \frac{X_{t}^{4}(v)}{t^{2}} m(\mathrm{~d} v)
$$

it suffices to prove that $\sup _{t>0} \int t^{-\frac{1}{2} s} X_{t}^{s}(v) m(\mathrm{~d} v)<+\infty$ for any $s>0$. Recall that $X_{t}$ may be decomposed into $S_{N_{t}} \phi()+.A\left(g_{t}.\right)-A($.$) up to a bounded term;$ so, $X_{t}^{s}($.$) is bounded by \left(S_{N_{t}} \phi(.)\right)^{s}+A\left(g_{t} .\right)^{s}+A(.)^{s}+1$, up to a multiplicative constant and it suffices to check that

$$
\sup _{t>0} \int \frac{\left(S_{N_{t}(v)} \phi\right)^{s}(x)}{t^{\frac{1}{2} s}} m(\mathrm{~d} v)<+\infty
$$

Denote by $\tilde{N}_{t}$ the random variable $\sqrt{t}\left(N_{t} / t-1 / \nu(\ell)\right)$. Fix $k \in \mathbb{Z}$, set $k_{t}=[t / \nu(\ell)+k \sqrt{t}]$ and observe that, on the set $\left\{\widetilde{N}_{t}(v) \in[k, k+1[\}\right.$, the difference between the sums $S_{N_{t}} \phi$ and $S_{k_{t}} \phi$ is bounded by $M_{[\sqrt{t}+1]} \phi \circ T^{k_{t}}$. So, for any $s>0$, one has

$$
\int_{\left\{\tilde{N}_{t} \in[k, k+1[\}\right.}\left(S_{N_{t}} \phi\right)^{2 s} m(\mathrm{~d} v) \leq \int_{\left\{\tilde{N}_{t} \in[k, k+1[\}\right.}\left(\left(S_{k_{t}} \phi\right)^{2 s}+\left(M_{[\sqrt{t}+1]} \phi\right)^{2 s}\right) m(\mathrm{~d} v)
$$

up to a multiplicative constant depending only on $s$. By Lemmas III.10 and III.12, since $k_{t}=O(t)$ and $[\sqrt{t}+1]=O(\sqrt{t})$, it readily follows that

$$
\int_{\left\{\tilde{N}_{t} \in[k, k+1[\}\right.}\left(S_{N_{t}} \phi\right)^{2 s} m(\mathrm{~d} v)=O\left(t^{s}\right) .
$$

TOME $134-2006-\mathrm{N}^{\mathrm{O}} 1$ 
Consequently, one has

$$
\begin{aligned}
\int\left(S_{N_{t}} \phi\right)^{s} m(\mathrm{~d} v) & \leq \sum_{k \in \mathbb{Z}} \int_{\left\{\widetilde{N}_{t} \in[k, k+1[\}\right.}\left(S_{N_{t}} \phi\right)^{s} m(\mathrm{~d} v) \\
& \leq \sum_{k \in \mathbb{Z}} \sqrt{\nu\left(\widetilde{N}_{t} \in[k, k+1[)\right.}\left(\int_{\left\{\widetilde{N}_{t} \in[k, k+1[\}\right.}\left(S_{N_{t}} \phi\right)^{2 s} m(\mathrm{~d} v)\right)^{\frac{1}{2}} \\
& \leq t^{\frac{1}{2} s} \sum_{k \in \mathbb{Z}} \sqrt{\nu\left(\widetilde{N}_{t} \in[k, k+1[)\right.}
\end{aligned}
$$

up to a multiplicative constant depending only on $s$. Using the definition of $N_{t}$ and Lemma III.10, one easily checks that the variable $\widetilde{N}_{t}$ has finite and bounded moments of any orders; so the sum $\sum_{k \in \mathbb{Z}} \sqrt{\nu\left(\widetilde{N}_{t} \in[k, k+1[)\right.}$ is also finite and bounded uniformly in $t$.

\section{Schottky products with parabolic subgroups and the associated winding process}

From now on, we consider a group $\Gamma$ which is a Schottky product $\Gamma_{1} * \cdots * \Gamma_{L}$ with a parabolic subgroup $P$ as a Schottky factor. We will use the notation of section II. Note that, if $\Gamma_{1}=P$, the group $\Gamma$ is the Schottky product of the two factors $\Gamma_{1}$ and $\Gamma_{2} * \cdots * \Gamma_{L}$; so, without loss of generality, we will assume $\Gamma=P * G$ with $P$ a parabolic subgroup of $\operatorname{Iso}(X)$ and $G$ a discrete subgroup.

In this section, we will assume that the curvature is constant and equal to -1 , i.e. $X=\mathbb{H}^{n}$. Note that we will only use the fact that there exists a neigbourhood of the cusp $\mathcal{C}$ associated with $P$ which is isometric to the quotient of some horoball of $\mathbb{H}^{n}$ by $P$; the curvature may thus vary outside this cusp but this level of generalisation is not very important and might be confusing in the sequel.

IV-a. Parabolic subgroups of Iso $\left(\mathbb{H}^{n}\right)$. - We recall here some classical results about parabolic subgroups $P$ of $\operatorname{Iso}\left(\mathbb{H}^{n}\right)$ (see [31]) and give a precise description of their first homology group.

A discrete subgroup $P$ of $\operatorname{Iso}\left(\mathbb{H}^{n}\right)$ is parabolic if it fixes exactly one point on the boundary. Let $x_{P}$ be this fixed point. The inversion $s: \mathbf{x} \mapsto x_{P}+$ $2 \mathbf{x}-x_{P} /\left|\mathbf{x}-x_{P}\right|^{2}$ sends the point $x_{P}$ to $\infty$ in the upper half-space model $\mathbb{H}^{n}=\left\{x+x^{\prime} x_{P} ; x \in x_{P}^{\perp}, x^{\prime}>0\right\}$ of the hyperbolic space (that we shall identify with $\mathbb{R}^{n-1} \times \mathbb{R}^{+}$). In this model, any element $p$ of $P$ acts on the boundary component $\mathbb{R}^{n-1}$ as an euclidean isometry, the restriction to $\mathcal{S}^{n-1}-\left\{x_{P}\right\}$ of each element $p$ in $P$ may thus be decomposed as

$$
p=s^{-1} T_{p} R_{p} s
$$

BULLETIN DE LA SOCIÉtÉ MATHÉMATIQUE DE FRANCE 
where $R_{p}$ is a rotation of $\mathbb{R}^{n-1}$ and $T_{p}$ a translation of $\mathbb{R}^{n-1}$. The vector of the translation will be denoted $\vec{\tau}_{p}$. The relation for large $p$ 's between the euclidean norm $\left|\vec{\tau}_{p}\right|$ and the dilatation factor of $p$ is described in the following lemma:

LEMMA IV.1. - Let $P$ be a discrete parabolic group of $\mathbb{H}^{n}$, with fixed point $x_{P}$. Then for each point $x \in \mathcal{S}^{n-1}-\left\{x_{P}\right\}$, the product $\left|p^{\prime}(x)\right| \cdot\left|\vec{\tau}_{p}\right|^{2}$ converges towards $4 /\left|x-x_{P}\right|^{2}$ as $p$ goes to infinity in $P$. This convergence is uniform on compact subsets of $\mathcal{S}^{n-1}-\left\{x_{P}\right\}$.

Proof. - The parabolic element $p \in P$ satisfies $\left|p^{\prime}\left(x_{P}\right)\right|=1$. By the meanvalue relation 2 , we get $\left|p^{\prime}(x)\right|=\left|p \cdot x-x_{P}\right|^{2} /\left|x-x_{P}\right|^{2}$. Now

$$
\left|p \cdot x-x_{P}\right|=\frac{2}{\left|s p \cdot x-x_{P}\right|}=\frac{2}{\left|R_{p}(s x)+\vec{\tau}_{p}-x_{P}\right|}
$$

and $\left|\vec{\tau}_{p}\right| \cdot\left|p \cdot x-x_{P}\right|$ converges uniformly towards 2 on compact sets in the hyperplane orthogonal to $x_{P}$. The lemma follows.

Note that the group $P$ acts on $\mathbb{R}^{n-1}$ as a Bieberbach's group; in particular it contains a finite index and normal subgroup $Q$ which acts on $\mathbb{R}^{n-1}$ as an abelian group generated by $k$ independent translations. The integer $k$ is called the rank of the parabolic group $P$.

The first homology group $H_{1}(P, \mathbb{Z})=P /[P, P]$ is the product of a finite group and the free abelian group $\mathbb{Z}^{\kappa}$ where $\kappa=b_{1}(P)$ is the first Betti number of $P$.

In dimension $n=2$ and $n=3$ all (orientation-preserving) Bieberbach groups of $\mathbb{R}^{n-1}$ are commutative and thus $b_{1}(P)$ coincides with the rank of $P$. This is not true in higher dimension: the group in $\mathbb{R}^{3}$ generated by $x \mapsto$ $\operatorname{diag}(-1,-1,1) x+e_{3}$ and $x \mapsto x+e_{2}$ is a Bieberbach group with rank 2 and first Betti number equal to 1 . In general, $b_{1}(P)$ is less than or equal to the rank of $P$.

The following lemma, which provides an explicit set of representatives of $P$ $\bmod Q$, will be helpful in order to compute the homology class of an arbitrary element in $P$.

LEMma IV.2. - Let $k$ be the rank of $P$ and $\kappa \leq k$ its first Betti number. Then there exists a finite set $\left\{q_{1}, \ldots, q_{k}\right\}$ of independent translations of $Q$, with $q_{\kappa+1}, \ldots, q_{k} \in Q \cap[P, P]$ and a finite set $P_{0}$ in $P$ such that any element $p \in P$ can be uniquely written as

$$
p=q_{1}^{n_{1}} \cdots q_{\kappa}^{n_{\kappa}} q_{\kappa+1}^{n_{\kappa+1}} \cdots q_{k}^{n_{k}} \alpha
$$

for some $n_{1}, \ldots, n_{k} \in \mathbb{Z}$ and $\alpha \in P_{0}$.

Proof. - Since the abelian subgroup $Q$ is of finite index in $P$, its image $[Q]$ by the Hurewicz homomorphism [.] : $P \rightarrow H_{1}(P, \mathbb{Z})$ has finite index in $\mathbb{Z}^{\kappa}$. We choose a basis $\left\{c_{1}, \ldots, c_{\kappa}\right\}$ of $\mathbb{Z}^{\kappa}$ and integers $d_{1}, \ldots d_{\kappa}$ such that $\left\{d_{1} c_{1}, \ldots, d_{\kappa} c_{\kappa}\right\}$ forms a basis of $[Q]$. This allows us to choose elements 
$p_{1}, \ldots p_{\kappa}$ in $P$ such that $\left[p_{i}\right]=c_{i}$ and $q_{1}, \ldots q_{\kappa}$ in $Q$ such that $\left[q_{i}\right]=d_{i} c_{i}$ and one may complete the family $\left\{q_{1}, \ldots q_{\kappa}\right\}$ with $k-\kappa$ independent translations in such a way the abelian group $Q_{0}$ generated by $q_{1}, \ldots, q_{k}$ has finite index in $Q$ (the elements $q_{\kappa+1}, \ldots, q_{k}$ belong to $\left.[P, P]\right)$. The homology class of any $p$ in $P$ may be written in the form

$$
[p]=\sum n_{i} d_{i} c_{i}+\sum m_{i} c_{i}
$$

for some integers $n_{i} \in \mathbb{Z}$ and $m_{i} \in\left\{0, \ldots, d_{i}-1\right\}$. In particular $p$ has the same homology class as $q_{1}^{n_{1}} \cdots q_{\kappa}^{n_{\kappa}} p_{1}^{m_{1}} \cdots p_{\kappa}^{m_{\kappa}}$, so there exists $p^{\prime} \in[P, P]$ such that

$$
p=q_{1}^{n_{1}} \cdots q_{\kappa}^{n_{\kappa}} p_{1}^{m_{1}} \cdots p_{\kappa}^{m_{\kappa}} p^{\prime} .
$$

Observe now that the subgroup $Q_{0} \cap[P, P]$ has finite index in $[P, P]$ since $[P, P] / Q_{0} \cap[P, P]$ is isomorphic to $Q_{0}[P, P] / Q_{0}$. By choosing a set $f_{1}, \ldots, f_{d}$ of representatives of $[P, P]$ mod $Q_{0} \cap[P, P]$, setting

$$
P_{0}=\left\{p_{1}^{m_{1}} \cdots p_{\kappa}^{m_{\kappa}} f_{j} ; 0 \leq m_{i}<d_{i}-1,1 \leq j \leq d\right\}
$$

and using the fact that $Q_{0}$ is abelian, we get the lemma.

Note that $[Q]$ is a finite index subgroup of the free abelian part of $H_{1}(P, \mathbb{Z})$. One may thus modify the decomposition of $H_{1}(P, \mathbb{Z})$ as the product of a finite group and a free abelian part $\mathbb{Z}^{\kappa}$ in such a way that this free abelian part is equal to $[Q]$; consequently if $p \in P$ is decomposed as $p=q_{1}^{n_{1}} \ldots q_{\kappa}^{n_{\kappa}} q_{\kappa+1}^{n_{\kappa+1}} \ldots q_{k}^{n_{k}} \alpha$ with $n_{i} \in \mathbb{Z}$ and $\alpha \in P_{0}$, the free part of its homology classes is equal to $[q]=$ $\left(n_{1}, \ldots, n_{\kappa}\right)$.

Notation. - In the sequel, the translation vector of each generator $q_{i}$ will be denoted by $\vec{\tau}_{i}$ and, for any $\bar{n}=\left(n_{1}, \ldots, n_{\kappa}\right) \in \mathbb{Z}^{\kappa}$, one sets

$$
\vec{\tau}_{\bar{n}}=n_{1} \vec{\tau}_{1}+\cdots+n_{\kappa} \vec{\tau}_{\kappa} .
$$

Using the $\delta$-conformality of the Patterson measure $\sigma$ one may describe precisely the behavior of the measure $\nu$ in the neighbourhood of the fixed point $x_{P}$. More precisely, Lemma IV.1 leads to the asymptotic behavior of the sequence $\left(\nu\left(p \Lambda_{G}\right)\right)_{p \in P}$ as $p$ goes to infinity in $P$. The following lemma gives in fact precise estimates of $\nu\left(\Lambda_{\bar{n}}\right), \bar{n} \in \mathbb{Z}^{\kappa}$, where $\Lambda_{\bar{n}}=\bigcup_{p /[p]=\bar{n}} p \cdot \Lambda_{G}$.

Lemma IV.3. - For any $\bar{n}=\left(n_{1}, \ldots, n_{\kappa}\right) \in \mathbb{Z}^{\kappa}$, denote by $|\bar{n}|$ the euclidean norm of the translation vector $\vec{\tau}_{\bar{n}}$ and let $\vec{n}=\vec{\tau}_{\bar{n}} /|\bar{n}|$. One has $\nu\left(\Lambda_{\bar{n}}\right)=\nu\left(\Lambda_{-\bar{n}}\right)$; furthermore, there exist a compact subset $K_{0} \subset \mathbb{R}^{*+}$ and, for any $\bar{n} \in \mathbb{Z}^{\kappa}$, a constant $C_{\vec{n}}$ in $K_{0}$ such that

$$
\nu\left(\Lambda_{\bar{n}}\right) \sim \frac{C_{\vec{n}}}{|\bar{n}|^{2 \delta-(k-\kappa)}} \quad \text { as } \quad \bar{n} \rightarrow+\infty \text { in } \mathbb{Z}^{\kappa} .
$$

Proof. - The fact that $\bar{\nu}$ is $\bar{T}$-invariant implies

$$
\nu\left(p \Lambda_{G}\right)=\bar{\nu}\left(\Lambda_{G} \times p \Lambda_{G}\right)=\bar{\nu}\left(p^{-1} \Lambda_{G} \times \Lambda_{G}\right) ;
$$

BULletin DE LA SOCiÉtÉ MATHÉmATiQUe DE FRANCE 
it readily follows $\nu\left(p \Lambda_{G}\right)=\nu\left(p^{-1} \Lambda_{G}\right)$ since $\bar{\nu}$ is invariant under the map $(x, y) \mapsto(y, x)$. The first property of the lemma follows, summing over $p \in P$ with $[p]=\bar{n}$.

Furthermore, for any $p \in P$ one has

$$
\begin{aligned}
\nu\left(p \Lambda_{G}\right) & =\int_{p \Lambda_{G}} h(x) \sigma(\mathrm{d} x) \\
& =\int_{\Lambda_{G}} h(p x)\left|p^{\prime}(x)\right|^{\delta} \sigma(\mathrm{d} x)=\int_{\Lambda_{G} \times \Lambda_{G}} \frac{\left|p^{\prime}(x)\right|^{\delta}}{|y-p x|^{2 \delta}} \sigma(\mathrm{d} x) \sigma(\mathrm{d} y)
\end{aligned}
$$

and so $\left|\vec{\tau}_{p}\right|^{2 \delta} \nu\left(p \Lambda_{G}\right) \rightarrow C=\left(\int_{\Lambda_{G}} 2^{\delta} /\left|x-x_{P}\right|^{2 \delta} \sigma(\mathrm{d} x)\right)^{2}$ as $p \rightarrow+\infty$ in $P$.

Recall that for any $p \in P$, the translation vector of $p$ is denoted by $\vec{\tau}_{p}$; if $p$ admits the decomposition $p=q_{1}^{n_{1}} \cdots q_{k}^{n_{k}} \alpha$, one has

$$
\left|\vec{\tau}_{p}\right|=\left|\vec{\tau}_{\bar{n}}+\sum_{\kappa+1}^{k} \vec{\tau}_{i}\right|(1+o(\bar{n}))
$$

uniformly in $\alpha$ since $P_{0}$ is finite. So, for any $\bar{n} \in \mathbb{Z}^{\kappa}$, one has

$$
\nu\left(\Lambda_{\bar{n}}\right)=\sum_{\alpha \in P_{0}} \sum_{n_{\kappa+1}, \ldots, n_{k} \in \mathbb{Z}} \frac{C}{\left|\vec{\tau}_{\bar{n}}+\sum_{i=\kappa+1}^{k} n_{i} \vec{\tau}_{i}\right|^{2 \delta}}(1+o(\bar{n})) .
$$

Denote by $|\Delta|$ the $(k-\kappa)$-volume of the simplex associated with $\vec{\tau}_{\kappa+1}, \ldots, \vec{\tau}_{k}$. One obtains

$$
\nu\left(\Lambda_{\bar{n}}\right) \sim \sharp P_{0} \frac{1}{|\Delta| \cdot|\bar{n}|^{2 \delta-(k-\kappa)}} \int_{V^{\prime \prime}} \frac{\mathrm{d} x}{|\vec{n}+x|^{2 \delta}} \quad \text { as } \quad \bar{n} \rightarrow+\infty,
$$

where $V^{\prime \prime}$ denotes the $(k-\kappa)$-dimensional space $\mathbb{R} \vec{\tau}_{\kappa+1} \oplus \cdots \oplus \mathbb{R} \vec{\tau}_{k}$. This proves the lemma with $C_{\vec{n}}=\left(\sharp P_{0} /|\Delta|\right) \int_{V^{\prime \prime}} \mathrm{d} x /\left(|\vec{n}+x|^{2 \delta}\right)$.

IV-b. The transfer operators $\mathcal{L}_{\boldsymbol{z}, \boldsymbol{u}, \boldsymbol{v}}$ - - We have constructed a dynamical system $(\bar{\Lambda}, \bar{T}, \bar{\nu})$ isomorphic to a symbolic space of bi-infinite sequences such that the geodesic flow restricted to $\Omega$ is a suspension of it with the ceiling function $\ell$. The projection $p: \bar{\Lambda} \rightarrow \Lambda$ on the second coordinate induces a factor $(\Lambda, T, \nu)$ of the dynamical system $(\bar{\Lambda}, \bar{T}, \bar{\nu})$.

In this section we study the family of transfer operators associated with the dynamical system $(\Lambda, T, \nu)$, a function $\mathbf{f}$ defined on $\Lambda$ and also the fonction $H_{P}$ defined by

$$
\forall \bar{n} \in \mathbb{Z}^{\ell}-\{0\}, \quad H_{P}(x)= \begin{cases}\bar{n} & \text { if } x \in \Lambda_{\bar{n}}, \\ 0 & \text { otherwise. }\end{cases}
$$

The function $\mathbf{f}$ is devoted to the process $X_{t}$ and $H_{P}$ to $Y_{t}$; we will precise a bit latter some conditions about the regularity of the function $\mathbf{f}$.

Let $C(\Lambda(\Gamma))$ be the space of continuous functions from $\Lambda_{\Gamma}$ to $\mathbb{C}$ with the norm of uniform convergence $|\cdot|_{\infty}$. For any complex number $z$, any real $u$ and

TOME $134-2006-\mathrm{N}^{\mathrm{O}} 1$ 
any element $v$ of the $\kappa$-dimensional torus $\mathbb{T}^{\kappa}$, canonically identified with the dual group of $\mathbb{Z}^{\kappa}$, we consider the operator $\mathcal{L}_{z, u, v}$ defined formally by

$$
\mathcal{L}_{z, u, v} \varphi(x)=\sum_{T y=x} \mathrm{e}^{-z \ell(y)+i u \mathbf{f}(y)+i\left\langle v \mid H_{P}(y)\right\rangle} \varphi(y) .
$$

Setting $[\gamma]=0$ when $\gamma \in G$, one may thus write

$$
\mathcal{L}_{z, u, v} \varphi(x)=\sum_{\gamma \in P^{*} \cup G^{*}} \mathrm{e}^{-z b(\gamma, x)+i u \mathbf{f}(\gamma \cdot x)+i\langle v \mid[\gamma]\rangle} \varphi(\gamma \cdot x) .
$$

This implies in particular that $\mathcal{L}_{z, u, v} \varphi(x)$ is finite as soon as $\Re(z)>$ $\max \left(\delta_{P}, \delta_{G}\right)$ and in particular for $\Re(z)=\delta_{\Gamma}$ by the critical gap hypothesis.

As in the previous section, we will consider the restriction of $\mathcal{L}_{z, u, v}$ to the space $L_{\alpha}$ of $\alpha$-Hölder continuous functions on $\Lambda(\Gamma)$, defined in Subsection III-c.

For any $(z, u, v) \in \mathbb{C} \times \mathbb{R} \times \mathbb{T}^{\kappa}$ and $\gamma \in P^{*} \cup G^{*}$, we consider the weight functions

$$
w_{z, u, v}(\gamma, .)=\mathrm{e}^{-z b(\gamma, .)+i u \mathbf{f}(\gamma \cdot)+i\langle v \mid[\gamma]\rangle} .
$$

Assume now that $\mathbf{f}$ satisfies the condition of Proposition III.8; as in Lemma III.3, one may check that the weights $w_{z, u, v}$ belong to $L_{\alpha}$ and that the set $\left\{\mathrm{e}^{\Re(z) d(\mathbf{o}, \gamma \mathbf{o})} w_{z, u, v}(\gamma,.) ; \gamma \in P^{*} \cup G^{*}\right\}$ is bounded in $L_{\alpha}$. By the critical gap property, it follows that the operators $\mathcal{L}_{\delta, u, v}$ act continuously on $L_{\alpha}$. The following proposition gives a precise description of the spectrum of $\mathcal{L}_{\delta, u, v}$ on $L_{\alpha}$ when $(u, v)$ is close to 0 ; its proof is based on the description of the spectrum of $\mathcal{L}_{\delta}$ (see Proposition III.4) and on the fact that the map $(u, v) \mapsto \mathcal{L}_{\delta, u, v}$ is continuous from $\mathbb{R} \times \mathbb{T}^{\kappa}$ to the space $\mathcal{L}\left(L_{\alpha}\right)$.

Proposition IV.4. - Fix $0<\alpha \leq 1$ and let $\rho(u, v)$ be the spectral radius of the transfer operator $\mathcal{L}_{\delta, u, v}$ on $L_{\alpha}$. Then, one has $\rho(0,0)=1$, the eigenvalues 1 and -1 are simple, with eigenfunctions $h$ and $h\left(1_{\Lambda_{G}}-1_{\Lambda_{P}}\right)$ respectively, and the rest of the spectrum of $\mathcal{L}_{\delta, 0,0}$ is included in a disk of radius $<1$.

Furthermore, there exists a neigbourhood $U_{0}$ of $(0,0)$ in $\mathbb{R} \times \mathbb{T}^{\kappa}$ and $\left.\rho_{0} \in\right] 0,1[$ such that, for any $(u, v) \in U_{0}$,

- $\rho(u, v)>\rho_{0}$ and $\mathcal{L}_{\delta, u, v}$ has two simple eigenvalues $\lambda(u, v)$ and $\lambda^{-}(u, v)$ closed to 1 and -1 respectively,

- the rest of the spectrum of $\mathcal{L}_{\delta, u, v}$ is included in a disc of radius $\rho_{0}$.

In fact, by the theory of perturbation, the functions $(u, v) \mapsto \lambda(u, v)$ and $(u, v) \mapsto \mathcal{L}_{\delta, u, v}$ have the same regularity, whose type is described in the following proposition, which extends previous results (see [2], [18]) settled in a less general situation. We denote by $\left(\vec{\tau}_{1}^{*}, \ldots, \vec{\tau}_{\kappa}^{*}\right)$ the dual basis of $\left(\vec{\tau}_{1}, \ldots, \vec{\tau}_{\kappa}\right)$, by $|v|$ the euclidean norm of the vector $v_{1} \vec{\tau}_{1}^{*}+\cdots+v_{\kappa} \vec{\tau}_{\kappa}^{*}$ for any $v=\left(v_{i}\right)_{1 \leq i \leq \kappa} \in \mathbb{T}^{\kappa}$, and by $\vec{v}$ the unit vector $v /|v|$, when $v \neq 0$. 
Proposition IV.5. - The application $(u, v) \mapsto \lambda(u, v)$ is continuous on $U_{0}$, and its behavior near $(0,0)$ is the following one, up to terms of greater order:

$$
\lambda(u, v)= \begin{cases}1-\frac{1}{2} \sigma_{\mathbf{f}}^{2} u^{2}-K(\vec{v})|v|^{2 \delta-k} & \text { if } \delta<1+\frac{1}{2} k \\ 1-\frac{1}{2} \sigma_{\mathbf{f}}^{2} u^{2}+K(\vec{v})|v|^{2} \log |v| & \text { if } \delta=1+\frac{1}{2} k \\ 1-Q(u, v) & \text { if } \delta>1+\frac{1}{2} k\end{cases}
$$

where

- $\sigma_{\mathbf{f}}^{2}$ is the asymptotic variance associated with $\mathbf{f}$, which vanishes if and only if $\mathbf{f}$ is a coboundary in $L_{\alpha}$;

- $K(\vec{v})$ is a strictly positive real depending only on $\vec{v}$;

- $Q$ is a positive quadratic form on $\mathbb{R}^{1+\kappa}$, whose restriction to $\mathbb{R}^{\kappa}$ is equal to $2 K(\vec{v})|v|^{2}$.

REMARK 1. - In fact, $K$ lives in a compact subset of $\mathbb{R}^{*+}$ since the function $\vec{v} \mapsto K(\vec{v})$ is continuous on the unit ball of $V^{\prime}=\mathbb{R} \vec{\tau}_{1} \oplus \cdots \oplus \mathbb{R} \vec{\tau}_{\kappa}$.

REMARK 2. - It is of interest to decide whether the quadratic form $Q$ is degenerated or not, but this question needs further assumptions; in particular if $\mathbf{f}$ coincides with one coordinate of $H_{P}$, the form $Q$ is trivially degenerated. Nevertheless, the restriction $v \mapsto Q(0, v)$ is positive definite on $\mathbb{R}^{\kappa}$. The important fact in the previous proposition is that the parameters $u$ and $v$ appear separately in the local expansion of $\lambda(u, v)$, when $\delta \leq 1+\frac{1}{2} k$ since in this case the functions $H_{i}, 1 \leq i \leq \kappa$ are not square integrable; this is this phenomenom which explains the asymptotic independance of $X_{t}$ and $Y_{t}$ announced in the introduction.

Proof of Proposition IV.5. - We have different cases to consider.

- First case: $\delta \leq 1+\frac{1}{2} k$.

a) In this case $\sum_{p \in P}\left|\vec{\tau}_{p}\right|^{2}\left\|w_{\delta}(p, .)\right\|_{\alpha}=+\infty$ and the function $(u, v) \mapsto \mathcal{L}_{\delta, u, v}$ is not $C^{2}$ on $\mathbb{T}^{\kappa+1}$. Nevertheless, the function $u \mapsto \mathcal{L}_{\delta, u, 0}$ is always $C^{\infty}$ and, by a similar argument than in the previous section, there exists $\sigma_{\mathbf{f}} \geq 0$ (wich vanishes if and only if $\mathbf{f}$ is a coboundary in $\left.L_{\alpha}\right)$ such that $\lambda(u, 0)=1-\frac{1}{2} \sigma_{\mathbf{f}}^{2} u^{2}(1+o(u))$.

b) We now study the function $v \mapsto \lambda(0, v)$. We have

$$
\lambda(0, v)=\sigma\left(\mathcal{L}_{\delta, 0, v} h\right)+\sigma\left(\left(\mathcal{L}_{\delta, 0, v}-\mathcal{L}_{\delta}\right)\left(h_{0, v}-h\right)\right) .
$$

Moreover

$$
\begin{aligned}
\sigma\left(\mathcal{L}_{\delta, 0, v} h\right)-1 & =\nu\left(\mathrm{e}^{i\left\langle v, H_{P}\right\rangle}\right)-1=\sum_{\bar{n} \in \mathbb{Z}^{\kappa}} \nu\left(\Lambda_{\bar{n}}\right)\left(\mathrm{e}^{i\langle v, \bar{n}\rangle}-1\right) \\
& =2 \sum_{\bar{n} \in \mathbb{Z}^{\kappa}}(\cos \langle v, \bar{n}\rangle-1) \nu\left(\Lambda_{\bar{n}}\right) \quad \text { since } \nu\left(\Lambda_{\bar{n}}\right)=\nu\left(\Lambda_{-\bar{n}}\right) \\
& =-2 \sum_{\bar{n} \in \mathbb{Z}^{\kappa}-\{0\}} C_{\bar{n}} \frac{1-\cos \langle v, \bar{n}\rangle}{|\bar{n}|^{2 \delta-(k-\kappa)}}(1+\epsilon(\bar{n})) .
\end{aligned}
$$

TOME $134-2006-\mathrm{N}^{\mathrm{O}} 1$ 
Recall that $V^{\prime}=\mathbb{R} \vec{\tau}_{1} \oplus \cdots \oplus \mathbb{R} \vec{\tau}_{\kappa}$ and set $V_{t}^{\prime}=t \mathbb{Z} \vec{\tau}_{1} \oplus \cdots \oplus t \mathbb{Z} \vec{\tau}_{\kappa}$, where $t=|v|$ is the euclidean norm of the vector $v_{1} \vec{\tau}_{1}^{*} \cdots \oplus v_{\kappa} \vec{\tau}_{\kappa}^{*}$. Let $|\Delta|$ be the volume of the $\kappa$-simplex of $V^{\prime}$ defined by $\vec{\tau}_{1}, \ldots, \vec{\tau}_{\kappa}$.

When $\delta<1+\frac{1}{2} k$, one has, using the estimates of Lemma IV.3:

$$
\begin{aligned}
\sum_{\bar{n} \in \mathbb{Z}^{\kappa}-\{0\}} C_{\vec{n}} \frac{1-\cos \langle v, \bar{n}\rangle}{|\bar{n}|^{2 \delta-(k-\kappa)}} & =\sum_{\bar{n} \in \mathbb{Z}^{\kappa}-\{0\}} C_{\vec{n}} \frac{1-\cos \left\langle\sum_{i=1}^{\kappa} v_{i} \vec{\tau}_{i}^{*}, \sum_{i=1}^{\kappa} n_{i} \vec{\tau}_{i}\right\rangle}{|\bar{n}|^{2 \delta-(k-\kappa)}} \\
& =\frac{t^{2 \delta-k}}{|\Delta|} \sum_{\bar{n} \in \mathbb{Z}^{\kappa}-\{0\}} C_{\vec{n}} \frac{1-\cos \langle\vec{v}, t \bar{n}\rangle}{|t \bar{n}|^{2 \delta-(k-\kappa)}}|\Delta| t^{\kappa} \\
& =\frac{t^{2 \delta-k}}{|\Delta|} \sum_{x \in V_{t}^{\prime}-\{0\}} C_{\vec{x}} \frac{1-\cos \langle\vec{v}, x\rangle}{|x|^{2 \delta-(k-\kappa)}}|\Delta| t^{\kappa} \sim K(\vec{v}) t^{2 \delta-k}
\end{aligned}
$$

with $K(\vec{v})=(1 /|\Delta|) \int_{V^{\prime}} C_{\vec{x}} 1-\cos \langle\vec{v}, x\rangle /|x|^{2 \delta-(k-\kappa)} \mathrm{d} x$. Note that one has $0<K(\vec{v})<+\infty$ since $2 \delta-k>0$ and that $K(\vec{v})$ depends continuously on $\vec{v}$.

When $\delta=1+\frac{1}{2} k$, one has

$$
\begin{gathered}
\sum_{\bar{n} \in \mathbb{Z}^{\kappa}-\{0\}} C_{\vec{n}} \frac{1-\cos \langle v, \bar{n}\rangle}{|\bar{n}|^{2 \delta-(k-\kappa)}}=\sum_{\bar{n} \in \mathbb{Z}^{\kappa}-\{0\}} C_{\vec{n}} \frac{1-\cos \langle v, \bar{n}\rangle}{|\bar{n}|^{\kappa+2}} \\
=\frac{t^{2}}{|\Delta|} \sum_{\substack{x \in V_{t}^{\prime}-\{0\} \\
C_{\vec{x}}}} \frac{1-\cos \langle\vec{v}, x\rangle}{|x|^{\kappa+2}}|\Delta| t^{\kappa} \\
=\frac{t^{2}}{|\Delta|} \sum_{\substack{x \in V_{t}^{\prime} \\
|x|^{\prime} 1}} C_{\vec{x}} \frac{1-\cos \langle\vec{v}, x\rangle}{|x|^{\kappa+2}}|\Delta| t^{\kappa} \\
+\frac{t^{2}}{|\Delta|} \sum_{\substack{x \in V_{t}^{\prime} \\
0<|x|<1}} C_{\vec{x}} \frac{1-\cos \langle\vec{v}, x\rangle-\frac{1}{2}\langle\vec{v}, x\rangle^{2}}{|x|^{\kappa+2}}|\Delta| t^{\kappa}+\frac{t^{2}}{2|\Delta|} \sum_{\substack{x \in V_{t}^{\prime} \\
0<|x|<1}} C_{\vec{x}} \frac{\langle\vec{v}, x\rangle^{2}}{|x|^{\kappa+2}}|\Delta| t^{\kappa} .
\end{gathered}
$$

As $t \rightarrow 0$, the two first terms of this last sum behave like $C t^{2}$ for some constant $C>0$ and one has

$$
\begin{aligned}
\sum_{\substack{x \in V_{t}^{\prime} \\
0<|x|<1}} C_{\vec{x}} \frac{\langle\vec{v}, x\rangle^{2}}{|x|^{\kappa+2}} t^{\ell} & \sim \int_{\left\{x \in M^{\prime} /|x|>1 / t\right\}} C_{\vec{x}} \frac{\langle\vec{v}, x\rangle^{2}}{|x|^{\kappa+2}} \mathrm{~d} x \\
& \sim \int_{\left\{x \in V^{\prime} /|x|>1 / t\right\}} C_{\vec{x}} \frac{\langle\vec{v}, \vec{x}\rangle^{2}}{|x|^{\kappa}} \mathrm{d} x \sim K(\vec{v}) \log t
\end{aligned}
$$

where $K(\vec{v})>0$ depends continuously on $\vec{v}$.

Finally $\sigma\left(\mathcal{L}_{\delta, 0, v} h\right)=1-Q(v)(1+o(v))$ with $Q(v)=-2 K(\vec{v})|v|^{2} \log |v|$ if $\delta=1+\frac{1}{2} k$ and $Q(v)=2 K(\vec{v})|v|^{2 \delta-k}$ if $\delta<1+\frac{1}{2} k$.

BULLETIN DE LA SOCiÉTÉ MATHÉMATIQUe DE FRANCE 
It remains to control the term $\sigma\left(\left(\mathcal{L}_{\delta, 0, v}-\mathcal{L}_{\delta}\right)\left(h_{0, v}-h\right)\right)$. A classical argument in perturbation theory implies $\left\|h_{0, v}-h\right\|=O\left(\left\|\mathcal{L}_{\delta, 0, v}-\mathcal{L}_{\delta}\right\|\right)$ with

$$
\left\|\mathcal{L}_{\delta, 0, v}-\mathcal{L}_{\delta}\right\| \leq \sum_{p \in P^{*}}\left|\mathrm{e}^{i\langle v,[p]\rangle}-1\right| \cdot\left\|w_{\delta}(p, .)\right\| \leq C \sum_{p \in P^{*}} \frac{\left|\mathrm{e}^{i\langle v,[p]\rangle}-1\right|}{\left|\vec{\tau}_{p}\right|^{2 \delta}} .
$$

A similar argument than the one developed above leads to

$\left\|\mathcal{L}_{\delta, 0, v}-\mathcal{L}_{\delta}\right\| \leq C R(v) \quad$ with $\quad R(v)= \begin{cases}+|v|^{2 \delta-k} & \text { if } \frac{1}{2} k<\delta<\frac{1}{2}(k+1), \\ -|v| \log |v| & \text { if } \delta=\frac{1}{2}(k+1), \\ +|v| & \text { if } \delta>\frac{1}{2}(k+1) .\end{cases}$

Since $R(v)^{2}=o(Q(v))$ one gets finally $\lambda(0, v)=1+Q(v)(1+o(v))$.

c) In order to control the residual term, one decomposes $\lambda(u, v)-1$ into

$$
\lambda(0, v)-1+\lambda(u, 0)-1+A(u, v)
$$

with $A(u, v)=\lambda(u, v)-\lambda(0, v)-\lambda(u, 0)+1$; using $\mathcal{L}_{\delta, u, v}-\mathcal{L}_{\delta, u, 0}=\mathcal{L}_{\delta, 0, v}-\mathcal{L}_{\delta}$ one gets

$$
\begin{array}{r}
A(u, v)=\sigma\left(\left(\mathcal{L}_{\delta, u, v}-\mathcal{L}_{\delta}\right)\left(h_{u, v}-h_{u, 0}\right)\right)+\sigma\left(\left(\mathcal{L}_{\delta, u, v}-\mathcal{L}_{\delta, u, 0}\right)\left(h_{u, 0}-h\right)\right) \\
-\sigma\left(\left(\mathcal{L}_{\delta, 0, v}-\mathcal{L}_{\delta}\right)\left(h_{0, v}-h\right)\right)
\end{array}
$$

and so $|A(u, v)|=O\left(\left\|\mathcal{L}_{\delta, 0, v}-\mathcal{L}_{\delta}\right\|^{2}+\left\|\mathcal{L}_{\delta, u, 0}-\mathcal{L}_{\delta}\right\| \cdot\left\|\mathcal{L}_{\delta, v, 0}-\mathcal{L}_{\delta}\right\|\right)$. This achieves the control of the residual term.

- Second case: $\delta>1+\frac{1}{2} k$.

Under this condition the series $\sum_{p \in P}\left|\cdot \vec{\tau}_{p}\right|^{2 \delta} \cdot\left\|w_{\delta}(p, .)\right\|_{\alpha}$ is convergent and the function $(u, v) \mapsto \mathcal{L}_{\delta, u, v}$ is $C^{2}$. So is the function $(u, v) \mapsto \lambda(u, v)$ and we now calculate its derivates of order 1 and 2 . Let $h_{u, v}$ be the unique eigenfunction of $\mathcal{L}_{\delta, u, v}$ associated with $\lambda(u, v)$ and such that $\sigma\left(h_{u, v}\right)=1$; the equality $\mathcal{L}_{\delta,-u,-v} \bar{h}_{u, v}=\overline{\lambda(u, v)} \bar{h}_{u, v}$ implies $\bar{h}_{u, v}=h_{-u,-v}$ and $\bar{\lambda}(u, v)=\lambda(\delta,-u,-v)$. The derivates at 0 of the function $(u, v) \mapsto h_{u, v}$ are thus purely imaginary valued.

In order to simplify the notation, and only all around the case $\delta>1+\frac{1}{2} k$, we set $H=\left(H_{n}\right)_{0<n<\kappa}$ with $H_{0}=\mathbf{f}$ and $H_{P}=\left(H_{n}\right)_{1<n<\kappa}$, and we denote by $\partial_{0}$ the partial derivate with respect to $u$ and, for $1 \leq n \leq \kappa$, by $\partial_{n}$ the partial derivate with respect to $v_{n}$; since the derivates at $(0,0)$ of the function $(u, v) \mapsto h_{u, v}$ belong to $L_{\alpha}$ and are purely imaginary complex valued, there exists real valued functions $d_{0}, \ldots, d_{n}$ in $L_{\alpha}$ such that $\partial_{n} h_{u, v \mid(u, v)=0}=i d_{n} h$; we set $D=\left(\mathrm{d}_{n}\right)_{n}$. Differentiating the two sides of $\lambda(u, v)=\sigma\left(\mathrm{e}^{i\langle(u, v), H(.)\rangle} h_{u, v}\right)$ yields to

$\left(^{*}\right) \quad \partial_{n} \lambda(u, v)=i \sigma\left(\mathrm{e}^{i\langle(u, v), H(.)\rangle} H_{n} h_{u, v}\right)+\sigma\left(\mathrm{e}^{i\langle(u, v), H(.)\rangle} \partial_{n} h_{u, v}\right)$

which gives, letting $(u, v)=0$ :

$$
\partial_{n} \lambda(0,0)=i \sigma\left(H_{n} h\right)+i \sigma\left(\mathrm{d}_{n} h\right)
$$

TOME $134-2006-\mathrm{N}^{\mathrm{O}} 1$ 
Since $\sigma\left(h_{u, v}\right)$ is always equal to 1 , one has $\sigma\left(\mathrm{d}_{n} h\right)=0$. On the other hand, one has $\sigma\left(H_{0} h\right)=\nu(\mathbf{f})=0$ by hypothesis and

$$
\left(\sigma\left(H_{n} h\right)\right)_{1 \leq n \leq \kappa}=\sum_{p \in P^{*}}[p] \nu\left(p \Lambda_{G}\right)=0
$$

since $\nu\left(p \Lambda_{G}\right)=\nu\left(p^{-1} \Lambda_{G}\right)$ and $[p]=-\left[p^{-1}\right]$. Finally $\partial_{n} \lambda(0,0)=0$ for all $n$.

Let us now differentiate the two sides of $(*)$. We get, letting $(u, v)=0$ :

$$
\begin{aligned}
\partial_{n} \partial_{m} \lambda(0,0) & =-\sigma\left(H_{n} H_{m} h+H_{n} d_{m} h+d_{n} H_{m} h\right) \\
& =-\nu\left(H_{n} H_{m}+H_{m} d_{m}+d_{n} H_{m}\right) .
\end{aligned}
$$

We have to prove that the quadratic form $Q=\left(-\partial_{n} \partial_{m} \lambda(0,0)\right)$ is positive definite. If one differentiates the equality $\mathcal{L}_{\delta, u, v} h_{u, v}=\lambda(u, v) h_{u, v}$, one gets $\mathcal{L}_{\delta}\left(H_{n} h\right)=d_{n} h-\mathcal{L}_{\delta} d_{n} h$ since $\partial_{n} \lambda(0,0)=0 ;$ so

$\nu\left(H_{n} d_{m} \circ T\right)=\sigma\left(H_{n} h d_{m} \circ T\right)=\sigma\left(\left(\mathrm{d}_{n} h-\mathcal{L}_{\delta}\left(\mathrm{d}_{n} h\right)\right) d_{m}\right)=\nu\left(\mathrm{d}_{n}\left(\mathrm{~d}_{m}-d_{m} \circ T\right)\right)$

(here, we have used the fact that $\sigma((\phi \circ T) \psi)=\sigma\left(\phi \mathcal{L}_{\delta} \psi\right)$ for any functions $\phi$ and $\psi$ in $\left.\mathbf{L}^{2}(\Lambda, \sigma)\right)$.

Set $u=v_{0}$; the equality $\nu\left(H_{n} d_{m} \circ T\right)=\nu\left(\mathrm{d}_{n}\left(\mathrm{~d}_{m}-d_{m} \circ T\right)\right)$ implies

$$
-\sum_{0 \leq n, m \leq \kappa} \partial_{n} \partial_{m} \lambda(0,0) v_{n} v_{m}=\nu\left(\langle(u, v), H+D-D \circ T\rangle^{2}\right),
$$

so this quadratic form is positive. The fact that $Q(0, v)=2 K(\vec{v})|v|^{2}$ follows from the equality $\sigma\left(\mathcal{L}_{\delta, 0, v} h\right)=1-2 K(\vec{v})|v|^{2}(1+o(v))$, which can be be proved using a similar argument than the one developed above in the case $\delta=1+\frac{1}{2} k$. This achieves the proof of Proposition IV.5.

The main consequence of Proposition IV.5 is a kind a homogeneity property of the quantity $\lambda(\delta, u, v)-1$ with respect to the variables $u$ and $v$, described in the following

Corollary IV.6. - For any $n \geq 1$ denote by $k_{n}$ the transformation of $\mathbb{R}^{1+\kappa}$ defined by

$$
k_{n}(u, v)=\left(\frac{u}{\sqrt{n}}, \frac{v}{d(n)}\right) \quad \text { with } \quad d(n)= \begin{cases}n^{1 /(2 \delta-k)} & \text { if } \delta<1+\frac{1}{2} k \\ \sqrt{n \log n} & \text { if } \delta=1+\frac{1}{2} k \\ \sqrt{n} & \text { if } \delta>1+\frac{1}{2} k .\end{cases}
$$

Then, for any $(u, v) \in \mathbb{R}^{1+\kappa}$, one has

$$
\lim _{n \rightarrow+\infty}\left(n\left(\lambda\left(k_{n}(u, v)\right)-1\right)= \begin{cases}K(\vec{v})|v|^{2 \delta-k}+\frac{1}{2} \sigma_{\mathbf{f}}^{2} u^{2} & \text { if } \delta \leq 1+\frac{1}{2} k, \\ Q(u, v) & \text { if } \delta>1+\frac{1}{2} k .\end{cases}\right.
$$

BULlETIN DE LA SOCIÉtÉ MATHÉMATIQUE DE FRANCE 
IV-c. The process $\left(Y_{t}(v)\right)$ and its decomposition. - In this section, we explain how the winding process $\left(Y_{t}\right)$ associated with a closed 1-form supported on a neighbourhood of the $\operatorname{cusp} \mathcal{C}$ can be presented as a Birkhoff sum over the dynamical system $(\Lambda, T, \nu)$ that we have just constructed. This presentation is the analogous of Proposition III.6 concerning the process $\left(X_{t}\right)$. We first give a description of the cusp $\mathcal{C}$.

$I V$-c.1. The cusp. - Since we know that $\Gamma$ is geometrically finite, by the Margulis Lemma (see [31]), there exists a horoball $\mathcal{H}_{h}=\left\{\left(x, x^{\prime}\right) ; x^{\prime} \geq h\right\}$ centered at $x_{P}$ such that its images under $\Gamma$ are equal or pairwise disjoint. The quotient manifold $\mathcal{C}_{h}=\mathcal{H}_{h} / P$ is homeomorphic to the cylinder $\mathbb{R}^{n-1} \times$ $[h,+\infty] / P$ and imbeds isometrically in $M$ as a submanifold with boundary. We call $\mathcal{C}_{h}$ the cusp (of height $h$ ) associated with $P$.

For further convenience, we shall also choose the horoball $\mathcal{H}_{h}$ so that closed geodesics arcs in $M$ represented by elements of $G$ and starting from a suitable compact neighborhood of some fixed origin in $M$ completely avoid the cusp $\mathcal{C}_{h}$.

This is possible according to the following lemma:

Lemma IV.7. - For any $r>0$, there exist $h>0$ only depending on $r$ such that for any two points $\mathbf{x}$ and $\mathbf{y}$ in $\mathbb{H}^{n}$ at distance less than $r$ from the origin, and any element $g \in G$, the geodesic segment $[\mathbf{x}, g \cdot \mathbf{y}]$ do not intersect the union of the horoballs $\gamma \mathcal{H}_{h}, \gamma \in \Gamma$.

Proof. - Note that the geodesic segment $[\mathbf{x}, g \cdot \mathbf{y}]$ belongs to the $r$-neighborhood of the geodesic arc $[o, g \cdot o]$. It suffices to prove that there exists a horoball $\mathcal{H}_{h}$ such that $\bigcup_{\gamma \in \Gamma} \gamma \mathcal{H}_{h}$ do not intersect $\bigcup_{g \in G}[o, g \cdot o]$, since by shrinking it by an amount only depending on $r$, any translate of it will avoid the $r$-neighborhood of $\bigcup_{g \in G}[o, g \cdot o]$. So suppose such a horoball does not exist. This provides for any integer $n$ two elements $g_{n} \in G$ and $\gamma_{n}$ in $\Gamma$ such that $\left[o, g_{n} \cdot o\right] \cap$ $\gamma_{n}^{-1} \mathcal{H}_{n} \neq \varnothing$ or equivalently such that $\left[\gamma_{n} \cdot o, \gamma_{n} g_{n} \cdot o\right] \cap \mathcal{H}_{n} \neq \varnothing$. Write $\gamma_{n}$ as a product of elements in $P$ and in $G$. Any horoball being invariant under $P$, we may assume that the first letter of $\gamma_{n}$ belongs to $G$, so that both $\gamma_{n}$ and $\gamma_{n} g_{n}$ belongs to $G \cup G^{*} \Gamma$. But for such sequences, any accumulation point at infinity belongs to the closed set $F_{G}$. Then, the endpoints of the geodesics arcs $\left[\gamma_{n} \cdot o, \gamma_{n} g_{n} \cdot o\right]$ either remain in a compact region of the hyperbolic space or accumulate in the compact set $F_{G} \subset \mathbb{R}^{n-1}$. This contradicts the fact that these geodesics arcs can meet arbitrarily small horoballs centered at $x_{P}$. The lemma is proved.

Note that $D$ has compact closure in $\Lambda(\Gamma) \stackrel{\Delta}{\times} \Lambda(\Gamma)$, so that the base points of the unit vectors $v=(y, x, 0)$ with $(y, x) \in D$ are at distance less than $r$ from the origin, for some $r$. In the following, we shall choose $h$ so that the lemma is satisfied with this value of $r$. In particular, if $y \in \Lambda_{P}$ and $x \in g \Lambda_{P}$ for some $g \in G^{*}$ the base point of $(y, x, \ell(x))$ is at a distance less than $r$ from $g \cdot \mathbf{o}$;

TOME $134-2006-\mathrm{N}^{\mathrm{O}} 1$ 
so, the geodesic segment $\{(y, x, s), 0 \leq s<l(x)\}$ does not intersect the union $\bigcup_{\gamma \in \Gamma} \gamma \mathcal{H}_{h}$. If $y \in \Lambda_{G}$ and $x \in p \Lambda_{G}$ for some $p \in P^{*}$ and if $h$ is large enough the segment $\{(y, x, s) ; 0 \leq s<f(x)\}$ does not intersect the horoballs $\gamma \mathcal{H}_{h}, \gamma \notin P$; on the other hand, for all but finitely many $p \in P$, it intersects the horoball $\mathcal{H}_{h}$.

$I V$-c.2. Decomposition of the process $\left(Y_{t}\right)_{t}$. - Let us precise that we say that the form $\omega$ on $M$ is supported on a neighbourhood of $\mathcal{C}$ if $\omega_{\mathbf{x}}=0$ when $\mathbf{x} \in \mathcal{C}_{h}$ for $h$ large enough; in the same way, we say that $\omega$ is closed on the cusp if $\omega$ is closed on $\mathcal{C}_{h}$ for some sufficiently big $h$.

Such a form $\omega$ induces an element $[\omega]$ in the first cohomology group $H^{1}\left(\mathcal{C}_{h}, \mathbb{R}\right)$. By De Rham Theorem, there exists a family $\left\{\omega_{1}, \ldots \omega_{\kappa}\right\}$ of 1 -forms on the manifold $\mathcal{C}_{2 h}$ whose cohomology classes provide a basis of $H^{1}\left(\mathcal{C}_{2 h}, \mathbb{R}\right)$ such that $\int_{\left[q_{j}\right]} \omega_{i}=\delta_{i j}$ (the elements $\left[q_{j}\right]$ are the homology classes of the elements $q_{j}$ of $P$ given by Lemma IV.1).The decomposition $[\omega]=\sum_{i=1}^{\kappa} v_{i}\left[\omega_{i}\right]$ means that the form $\omega$ restricted to $\mathcal{C}_{2 h}$ is equal to $\sum v_{i} \omega_{i}$ plus an exact form on $\mathcal{C}_{2 h}$. By adding this exact term to $\omega_{1}$, we may (and shall) assume that $\omega-\sum v_{i} \omega_{i}$ vanishes on $\mathcal{C}_{2 h}$. The forms $\omega_{1}, \ldots, \omega_{\kappa}$ can then be extended to $M$ in such a way they vanish outside $\mathcal{C}_{h}$. Finally, we can write $\omega$ as

$$
\omega=\omega_{0}+\sum_{i=1}^{\kappa} v_{i} \omega_{i}
$$

where $\omega_{0}$ has compact support in $M$, and the $\omega_{i}$ are 1 -forms supported on $\mathcal{C}_{h}$ and closed on $\mathcal{C}_{2 h}$.

Consider now the process $Y_{t}: T^{1} M \rightarrow \mathbb{R}$ given by $Y_{t}(v)=\int_{\left[v_{0}, v_{t}\right]} \omega$ where for $t>0,\left[v_{0}, v_{t}\right]$ is the geodesic segment of length $t$ starting at $v \in T^{1} M$.

Proposition IV.8. - For $i=1, \ldots, \kappa$, denote by $H_{i}$ the function from $\Lambda$ to $\mathbb{R}$ with constant value $\int_{[p]} \omega_{i}$ on the element $p \Lambda_{G}$ of the partition of $\Lambda$ and by $\mathbf{H}$ the function $\mathbf{H}=\sum_{i=1}^{\kappa} v_{i} H_{i}$. Then there exists a Borel function $A$ : $D_{\ell} \rightarrow \mathbb{R}$ and a Hölder continuous function $\mathbf{h}: \Lambda \rightarrow \mathbb{R}$ such that for any $v=(y, x, r) \in \Lambda$ one has

$$
Y_{t}(v)=A\left(v_{t}\right)-A(v)+\sum_{k=0}^{N_{t}(v)-1} \mathbf{h}\left(T^{k} x\right)+\sum_{k=0}^{N_{t}(v)-1} \mathbf{H}\left(T^{k} x\right) .
$$

Moreover there exists a constant $C$ such that:

i) $|\mathbf{h}(x)| \leq C \ell(x)+C$ for any $x$ in $\Lambda$;

ii) $|\mathbf{h}(p \cdot x)-\mathbf{h}(p \cdot y)| \leq C|x-y|^{\frac{1}{2}}$ for any two points $x, y$ in $\Lambda_{G}$ and $p \in G$;

iii) $|\mathbf{h}(g \cdot x)-\mathbf{h}(g \cdot y)| \leq C|x-y|^{\frac{1}{2}}$ for any two points $x, y$ in $\Lambda_{P}$ and $g \in G$.

The proof is close to the one of Proposition III.6 with some adjustments we have to precise.

BULletin DE LA SOCiÉtÉ MATHÉmATiQUE DE FRANCE 
Observe that the $\Gamma$-invariance of the lift $\widetilde{\omega}$ of $\omega$ implies that the function $(y, x, r) \mapsto \widetilde{\omega}(y, x, r)$ is invariant under the transformation $T_{f}$. This allows us to write:

$$
\begin{aligned}
Y_{t}(v) & =\int_{0}^{t} \widetilde{\omega}(y, x, r+s) \mathrm{d} s \\
& =-\int_{0}^{r} \widetilde{\omega}(y, x, s) \mathrm{d} s+\int_{0}^{S_{n} \ell(x)} \widetilde{\omega}(y, x, s) \mathrm{d} s+\int_{S_{n} \ell(x)}^{t+r} \widetilde{\omega}(y, x, s) \mathrm{d} s \\
& =B\left(v_{t}\right)-B(v)+\sum_{k=0}^{n-1} \Omega\left(\bar{T}^{k}(y, x)\right)
\end{aligned}
$$

where we have set $B(v)=\int_{0}^{r} \tilde{\omega}(y, x, s) \mathrm{d} s$ and $\Omega(y, x)=\int_{0}^{\ell(x)} \widetilde{\omega}(y, x, s) \mathrm{d} s$ and $n \in \mathbb{N}$ is such that $S_{n} \ell(x) \leq r+t<S_{n+1} \ell(x)$. Recall now the decomposition $\omega=\omega_{0}+\sum_{i=1}^{\kappa} v_{i} \omega_{i}$. Since the 1-form $\omega_{0}$ has compact support, the function $\Omega_{0}(y, x)=\int_{0}^{f(x)} \widetilde{\omega}_{0}(y, x, s) \mathrm{d} s$ is bounded and Lispschitz continuous on $T^{1} M$. For such a function, there exists a measurable function $b_{0}: \bar{\Lambda} \rightarrow \mathbb{R}$ and some function $\mathbf{g}_{0}$ satisfying the properties i), ii), iii) of Proposition IV.8 such that

$$
\sum_{k=0}^{n-1} \Omega_{0}\left(\bar{T}^{k}(y, x)\right)=\sum_{k=0}^{n-1} \mathbf{g}_{0}\left(T^{k} x\right)+b_{0}\left(T^{n}(y, x)\right)-b_{0}(y, x) .
$$

Let us now look at the integrals $\Omega_{i}(y, x)=\int_{0}^{\ell(x)} \widetilde{\omega}_{i}(y, x, s) \mathrm{d} s$, for $1 \leq i \leq \kappa$, which give the contribution coming from the excursions in the cusp. Recall that, by the choice of $h$,(cf. Lemma IV.7), if $y \in \Lambda_{P}$ and $x \in g \Lambda_{P}$ for some $g \in G^{*}$, the geodesic segment $\{(y, x, s) ; 0 \leq s<\ell(x)\}$ does not intersect the union $\bigcup_{\gamma \in \Gamma} \gamma \mathcal{H}_{h}$; the above integrals are then equal to 0 in this case. Assume now that $y \in \Lambda_{G}$ and $x \in p \Lambda_{G}$ for some $p \in P^{*}$. Since $h$ is large enough, the segment $\{(y, x, s) ; 0 \leq s<\ell(x)\}$ does not intersect the horoballs $\gamma \mathcal{H}_{h} \neq \mathcal{H}$, but for all but finitely many $p \in P$, this segment intersects the horoball $\mathcal{H}_{h}$; let $\mathbf{x}_{0}$ (resp. $\mathbf{x}_{1}$ ) be the point where this segment enters (resp. leaves) $\mathcal{H}_{h}$. The hyperbolic distances between $\mathbf{x}_{0}$ and the base point of $(y, x, 0)$ and between $p \cdot \mathbf{x}_{0}$ and the base point of $(y, x, \ell(x))$ are bounded; that means that $\Omega_{i}(y, x)$ differs from the integral $\int_{\left[\mathbf{x}_{0}, p \cdot \mathbf{x}_{0}\right]} \widetilde{\omega}_{i}=\int_{[p]} \omega_{i}$ by a bounded Lipschitz term $G_{i}(y, x)$. This implies that

$$
\sum_{k=0}^{n-1} \Omega_{i}\left(\bar{T}^{k}(y, x)\right)=\sum_{k=0}^{n-1} H_{i}\left(T^{k} x\right)+\sum_{k=0}^{n-1} G_{i}\left(\bar{T}^{k}(y, x)\right) .
$$

Since $G_{i}$ is bounded and Lipschitz, a classical argument similar to the one developed in Section III-d, step 1, allows us to find measurable functions $b_{i}$ : $\bar{\Lambda} \rightarrow \mathbb{R}$ and functions $\mathbf{g}_{i}: \Lambda \rightarrow \mathbb{R}$ satisfying properties i), ii) and iii) above, and such that $G_{i}(y, x)=\mathbf{g}_{i}(x)+b_{i}\left(\bar{T}^{n}(y, x)\right)-b_{i}(y, x)$.

TOME $134-2006-\mathrm{N}^{\mathrm{O}} 1$ 
Finally, we obtain the expected decomposition of $Y_{t}(v)$ setting $A(y, x, s)=$ $B(y, x, s)+\sum_{i=0}^{\kappa} b_{i}(y, x)$ and $\mathbf{h}=\sum_{i=0}^{\kappa} \mathbf{g}_{i}$. This achieves the proof of Proposition IV.8.

IV-d. The convergence in law for the process $\left(\boldsymbol{Y}_{\boldsymbol{t}}\right)_{t} \cdot-$ We prove the following

THEOREM IV.9. - Let $\Gamma$ a discrete subgroup in $\mathrm{Iso}(\mathbb{H})$ which is a Schottky product and which satisfies the critical gap property. Assume that the manifold $M=\mathbb{H} / \Gamma$ contains a cusp $\mathcal{C}$ whose fundamental group $P=\pi_{1}(\mathcal{C})$ is a Schottky factor of $\Gamma$. We denote by $\delta$ the Hausdorff dimension of the limit set of $\Gamma$ and by $k$ the rank of the parabolic group $P$.

For a closed 1 -form $\omega$ supported on $\mathcal{C}$, the corresponding process $\left(Y_{t}, m\right)$ satisfies a limit theorem. The renormalising factor and the limit law depend on the values of the parameter $\alpha:=2 \delta-k$ as follows:

- if $\alpha<2, \quad d(t)=t^{1 / \alpha}$ and the limit law is a stable law of index $\alpha$;

- if $\alpha=2, \quad d(t)=\sqrt{t \log t}$ and the limit law is a normal law;

- if $\alpha>2, \quad d(t)=\sqrt{t}$ and the limit law is a normal law.

Furthermore, when $\alpha \leq 2$, the two processes $\left(X_{t}\right)_{t}$ and $\left(Y_{t}\right)_{t}$ are asymptotically independent.

Recall the decomposition

$$
Y_{t}(v)=\sum_{k=0}^{N_{t}(v)-1} \mathbf{h}\left(T^{k} x\right)+\sum_{k=0}^{N_{t}(v)-1} \mathbf{H}\left(T^{k} x\right)+R_{t}(v)
$$

where $\mathbf{h}$ satisfies the hypotheses of Proposition III.8 and the remainder term $R_{t}$ is of the form $A\left(g_{t} v\right)-A(v)$ up to an additive uniformly bounded Borel function.

To control the Birkhof sums relative to the function $H_{P}$, we will use the

Proposition IV.10. - Let $d(n)$ be the sequence defined in Corollary IV.6. Then, when $x$ is distributed among the law $\nu$, the sequence

$$
\left(\frac{1}{d(n)} \sum_{k=0}^{n-1} H_{P}\left(T^{k} x\right)\right)_{n}
$$

converges in law to a $\kappa$-dimensionnal stable law of index $\alpha=2 \delta-k$. More precisely, for any $v \in \mathbb{T}^{\kappa}$, one has

$$
\lim _{n \rightarrow+\infty} \int_{\Lambda} \exp \left(i\left\langle v \mid \frac{S_{n} H_{P}(x)}{d(n)}\right\rangle\right) \nu(\mathrm{d} x)= \begin{cases}\mathrm{e}^{-K(\vec{v})|v|^{2 \delta-k}} & \text { if } \delta \leq 1+\frac{1}{2} k \\ \mathrm{e}^{-K(\vec{v})|v|^{2}} & \text { if } \delta>1+\frac{1}{2} k .\end{cases}
$$

where $K(\vec{v})$ is a constant which lies in a compact subset of $\mathbb{R}^{*+}$ and depends continuously on $\vec{v}$. Furthermore, for any Borel function $\mathbf{f}$ satisfying hypotheses 
of Proposition III.8 and such that $\nu(\mathbf{f})=0$ and $\sigma_{\mathbf{f}}^{2} \neq 0$, one has, when $\delta \leq 1+\frac{1}{2} k$

$$
\lim _{n \rightarrow+\infty} \int_{\Lambda} \exp i\left(u \frac{S_{n} \mathbf{f}(x)}{\sqrt{n}}+\left\langle v \mid \frac{S_{n} H_{P}(x)}{d(n)}\right\rangle\right) \nu(\mathrm{d} x)=\mathrm{e}^{-\frac{1}{2} \sigma_{\mathrm{f}}^{2} u^{2}} \mathrm{e}^{-K(\vec{v})|v|^{2 \delta-k}} .
$$

The proof of this statement is based on the identity

$$
\int_{\Lambda} \exp \left(i\left\langle v \mid \frac{S_{n} H_{P}(x)}{d(n)}\right\rangle\right) \nu(\mathrm{d} x)=\int_{\Lambda} \mathcal{L}_{\delta, 0, v / d(n)}^{n} h(x) \sigma(\mathrm{d} x) .
$$

As in Proposition III.8, one may check that the dominant term of of $\sigma\left(\mathcal{L}_{\delta, 0, v}^{n} h\right)$ is $\lambda_{\delta, 0, v}^{n}$; Corollary IV.6 allows us to conclude. The last assertion of the Proposition is a direct consequence of the Proposition IV.5 and the Remark 2 which follows.

Corollary IV.11. - Let $\mathbf{H}=\sum_{i=1}^{\kappa} v_{i} H_{i}$ be the function associated with the closed form $\omega$ and defined in Proposition IV.8. Then the sequence $\left(1 / d(n) S_{n} \mathbf{H}(x)\right)_{n}$ converges in law to a non degenerated 1-dimensionnal stable law of index $\alpha=2 \delta-k$; in other words, for any $t \in \mathbb{R}$, one has

$$
\lim _{n \rightarrow+\infty} \int_{\Lambda} \exp \left(i t \frac{S_{n} \mathbf{H}(x)}{d(n)}\right) \nu(\mathrm{d} x)= \begin{cases}\mathrm{e}^{-A|t|^{2 \delta-k}} & \text { if } \delta \leq 1+\frac{1}{2} k \\ \mathrm{e}^{-A t^{2}} & \text { if } \delta>1+\frac{1}{2} k\end{cases}
$$

for some constant $A>0$ which depends on $\mathbf{H}$.

We now give the main steps of the proof of Theorem IV.9. The term $1 / d(t) R_{t}(v)$ is not relevant since it tends to 0 in probability. The control of the others terms is more delicate:

- When $\delta>1+\frac{1}{2} k$, the function $\mathbf{h}+\mathbf{H}$ is square integrable on $(\Lambda, \nu)$ and satisfies the conditions ii) and iii) of Proposition IV.8; this is in fact sufficient to apply Proposition III.8 (the same proof holds since the function $u \mapsto \mathcal{L}_{\delta, u}$ is also twice continuously differentiable for $\mathbf{f}=\mathbf{h}+\mathbf{H}$ ). The associated variance is $\neq 0$ since the function $\mathbf{h}+\mathbf{H}$ is not bounded. Now the fact that the number $N_{t}$ of terms in the above sum is a random variable can be treated as in the previous section.

- Assume now $\delta \leq 1+\frac{1}{2} k$. Since the term $\sum_{k=0}^{N_{t}(v)-1} \mathbf{h}\left(T^{k} x\right)$ satisfies the classical Central Limit Theorem and $d(t) \gg \sqrt{t}$ for such values of $\delta$, the process $\left(1 / d(t) \sum_{k=0}^{N_{t}(v)-1} \mathbf{h}\left(T^{k} x\right)\right)_{t}$ converges to 0 in probability. To look at the last term $\sum_{k=0}^{N_{t}(v)-1} \mathbf{H}\left(T^{k} x\right)$, set $n_{t}=[t / \nu(\ell)]$. By Corollary IV.6, the process $1 / d(t) \sum_{k=0}^{n_{t}-1} \mathbf{H}\left(T^{k} x\right)$ converges to a non degenerated stable law of in$\operatorname{dex} \alpha=2 \delta-k$; the control of the difference between the two sums $\sum_{k=0}^{N_{t}(v)-1}$ and $\sum_{k=0}^{n_{t}-1}$ may be lead as in the previous section, since $d(t) \gg \sqrt{t}$.

The asymptotic independance of $\left(X_{t}\right)_{t}$ and $\left(Y_{t}\right)_{t}$ when $\delta \leq 1+\frac{1}{2} k$ follows from the last assertion of Proposition IV.10.

TOME $134-2006-\mathrm{N}^{\mathrm{O}} 1$ 


\section{BIBLIOGRAPHY}

[1] Artin (E.) - Ein mechanisches System mit quasiergodishen Bahnen, p. 499-501, Collected papers, Addison Wesley, 1965.

[2] Babillot (M.) \& Peigné (M.) - Homologie des géodésiques fermées sur des variétés hyperboliques avec bouts cuspidaux, Ann. Sci. École Norm. Sup., t. 33 (2000), pp. 81-120.

[3] Beardon (A. F.) - The exponent of convergence of Poincaré series, Proc. London Math. Soc., t. 18 (1968), pp. 461-483.

[4] Bourdon (M.) - Structure conforme au bord et flot géodésique d'un CAT (-1)-espace, Enseign. Math., t. 118 (1995), pp. 63-102.

[5] Bowen (R.) - Equilibrium states and the ergodic theory of Anosov diffeomorphisms, in Lecture Notes in Mathematics, vol. 470, 1975.

[6] Bowen (R.) \& Series (C.) - Markov maps associated with Fuchsian groups, Publ. Math. IHÉS, t. 50 (1979), pp. 153-170.

[7] Conze (J.-P.) \& LE Borgne (S.) - Méthode de martingales et flot géodésique sur une surface de courbure constante négative, Ergo. Th. Dyn. Syst., t. 21 (2001), pp. 421-441.

[8] Dal’bo (F.), Otal (J.-P.) \& Peigné (M.) - Séries de Poincaré des groupes géométriquement finis, Israel J. Math., t. 118 (2000), pp. 109-124.

[9] Dal'Bo (F.) \& Peigné (M.) - Groupes du ping-pong et géodésiques fermées en courbure -1, Ann. Inst. Fourier, t. 46 (1996), pp. 755-799.

[10] Doeblin (W.) \& Fortet (R.) - Sur les chaînes à liaison complètes, Bull. Soc. Math. France, t. 65 (1937), pp. 132-148.

[11] Eberlein (P.) - Geodesic flows on negatively curved manifolds, Ann. of Math., t. 95 (1973), pp. 492-510.

[12] Enriquez (N.), Franchi (J.) \& Le JAN (Y.) - Central Limit Theorem for the geodesic flow associated with a Kleinian group, case $\delta>\frac{1}{2} d$, J. Math. Pures Appl., t. 80 (2001), pp. 153-175.

[13] EnRiquez (N.) \& LE JAN (Y.) - Statistic of the winding of geodesics on a Riemann surface with finite volume and constant negative curvature, Rev. Mat. Ibero., t. 13 (1997), pp. 377-401.

[14] FRANCHi (J.) - Asymptotic singular homology of a complete hyperbolic 3-manifold of finite volume, Proc. London Math. Soc., t. 79 (1999), pp. 451-480.

[15] Gel'fand (I. M.) \& Pyateckim-Sapiro (I. I.) - A theorem of Poincaré, Dokl. Akad. Nauk. SSSR, t. 127 (1959), pp. 490-493.

[16] Ghys (E.) \& DE LA HARPe (P.) - Sur les groupes hyperboliques d'après M. Gromov, in Progress in Math., vol. 83, Birkhaüser, 1988. 
[17] Guivarc'H (Y.) \& HaRdy (J.) - Théorèmes limites pour une classe de châ̂nes de Markov et applications aux difféomorphismes d'Anosov, Ann. Inst. Henri Poincaré, t. 24, pp. 73-98.

[18] Guivarc'H (Y.) \& LE JAN (Y.) - Asymptotic winding of the geodesic flow on modular surfaces and continuous fractions, Ann. Sci. École Norm. Sup., t. 26 (1993), pp. 23-50.

[19] Note rectificative: "Asymptotic winding of the geodesic flow on modular surfaces and continuous fractions", Ann. Sci. École Norm. Sup. (1996), pp. 811-814.

[20] Hennion (H.) - Sur un théorème spectral et son application aux noyaux Lipschitziens, Proc. Amer. Math. Soc., t. 118 (1993), pp. 637-634.

[21] Hennion (H.) \& Hervé (L.) - Limit theorem for Markov chains and stochastic properties of dynamical systems by quasi-compactness, in Lecture Notes in Math., vol. 1766.

[22] KaÏmanovitch (V.) - Invariant measures of the geodesic flow and measures at infinity on negatively curved manifolds, Ann. Inst. Henri Poincaré, t. 53 (1900), pp. 361-393.

[23] LALlEy (S.) - Closed geodesics in homology classes on surfaces of variable negative curvature, Duke Math. J., t. 58 (1989), pp. 795-821.

[24] Le Borgne (S.) - Principe d'invariance pour les flots diagonaux sur $\mathrm{SL}(d, \mathbb{R}) / \mathrm{SL}(d, \mathbb{Z})$, Ann. Inst. Henri Poincaré, t. 38 (2002), pp. 581-612.

[25] LE JAN (Y.) - The central Limit Theorem for the geodesic flow on non compact manifolds of constant negative curvature, Duke Math. J., t. 74 (1994), pp. 159-175.

[26] Maskitt (B.) - Kleinian Groups, Springer-Verlag, Berlin, 1988.

[27] Otal (J.-P.) \& Peigné (M.) - Principe variationnel et groupes Kleiniens, To appear in Duke Math. J.

[28] PARry (W.) \& Pollicott (M.) - Zeta functions and the periodic orbit stucture of hyperbolic dynamics, Astérisque (1990), pp. 187-188.

[29] Patterson (S. J.) - The limit set of a Fuchsian group, Acta Math., t. 136 (1976), pp. 241-273.

[30] Peigné (M.) - On the Patterson-Sullivan measure of some discrete groups of isometries, Israel J. Math., t. 133 (2003), pp. 77-88.

[31] Ratcliffe (J. G.) - Foundations of Hyperbolic Geometry, Springer Verlag, New York, 1994.

[32] Ratner (M.) - The Central Limit Theorem for geodesic flows on $n$ manifolds of negative curvature, Israel J. Math., t. 16 (1973), pp. 181-197.

[33] SERIEs (C.) - The modular surface and continued fractions, J. London Math. Soc., t. 31 (1985), pp. 69-80.

TOME $134-2006-\mathrm{N}^{\mathrm{O}} 1$ 
[34] Sinai (J. G.) - The Central Limit Theorem for geodesic flows on manifolds of constant negative curvature, Dokl. Akad. Nauk. SSSR, t. 1 (1960), pp. 983-987.

[35] Stratmann (B.) \& Velani (S. L.) - The Patterson measure for geometrically finite groups with parabolic elements, new and old, Proc. Lond. Math. Soc., t. 71 (1995), pp. 197-220.

[36] Sullivan (D.) - The density at infinity of a discrete group of hyperbolic motions, Publ. Math. IHÉS, t. 50 (1979), pp. 171-202. 